\title{
Factor Receptor (EGFR) system
}

7

8 Stefano Pascarelli ${ }^{1}$, Dalmira Merzhakupova ${ }^{1}$, Gen-Ichiro Uechi ${ }^{1}$, Paola Laurino $^{1 *}$

9

101 Okinawa Institute of Science and Technology Graduate University, 1919-1 Tancha, Onna-son, Okinawa 904-

110495 Japan.

12

13

14

$15 *$ Corresponding author

16 E-mail: paola.laurino@oist.jp

17 


\section{Abstract}

19 The Epidermal Growth Factor Receptor (EGFR) is a membrane-anchored tyrosine kinase that

20 is able to respond to multiple extra-cellular stimuli in a selective way. Previous studies have

21 pointed out that the modularity of this system could be carried out by ligand-induced

22 differences in the stability of the dimerized receptor. However, nobody has ever explored this

23 hypothesis by observing the effects of single-mutant ligands so far.

24 Herein, we generated single mutants after we identified positions responsible of inducing

25 functional divergence among paralog ligands using a newly developed approach. Then, we

26 assessed the mutants' effects on the receptor by employing a combination of molecular

27 dynamics and experimental techniques. Although having comparable binding affinities for

28 EGFR to the wild type, the mutants induced different responses at both the receptor and the

29 cellular level. This study shows for the first time that a functional transition in EGFR can be

30 imparted with as little as one targeted mutation on the ligand. These results also support the

31 theory of biased signaling in the tyrosine kinase receptor system.

33 Abbreviations

34 EGF, Epidermal Growth Factor

35 EGFR, Epidermal Growth Factor Receptor

36 PPI, Protein-Protein Interaction

37 MSA, Multiple Sequence Alignment

38 MSTA, Multiple STructural Alignment

39 ITC, Isothermal Titration Calorimetry

40 DMEM, Dulbecco's Modified Eagle Medium

41 ECD, Extra Cellular Domain

42 WT, Wild Type 
CD, circular dichroism

\section{Introduction}

The Epidermal Growth Factor (EGF)-like domain ligand-receptor signaling system is involved in many biological events in multicellular organisms (1). This system is constituted by one receptor (EGFR) and seven distinct peptide ligands (EGF, HBEGF, EPGN, BTC, EREG, AREG, TGFA). Upon binding to the receptor, these ligands can activate multiple intracellular downstream pathways through an interaction network with a bow-tie structure (2). For all ligands, binding induces a transition from monomer or inactive dimer to an active dimer state $(3,4)$. However, different ligands are able to promote divergent outcomes, even at saturating concentrations; thus, the mechanism responsible of the modular downstream pathway activation is independent of ligand affinity or potency, and likely encompasses intrinsic effects (5). It is well known that the EGFR system play a key role in cancer development. In particular, some studies have shown that overexpression of EGFR or its ligands may induce different types of cancer (6). A better understanding of the interaction between EGFR and its ligands could lead to the development of targeted therapies (7).

62 Protein-protein interactions (PPIs), such as that between EGFR and its ligands, are some of the most interesting and well-studied examples of molecular co-evolution in biological systems.

64 These interactions are sometimes defined by one part (receptor) that binds several counterparts (ligands). In these instances, receptors and ligands experience different selective constraints, where receptors tend to evolve more slowly due to the necessity of binding multiple ligands

67 (8). Furthermore, paralogs, proteins related by a duplication event, are less likely to retain the 
same function as orthologs, proteins related by a speciation event (9). Then, paralogous ligands rather than the receptor are a good candidate to test the functional divergence in the biased signaling system. However, this approach is susceptible to indirect factors that get into play. For example, not all ligands might have the same binding selectivity, thus resulting in the activation of unrelated pathways. In this work, we decided to test single mutants of one ligand, EGF, by identifying and modifying sites responsible for a divergent function among paralog ligands. In recent years, different experimental techniques have been developed to define the effects of single mutant proteins at the cellular level (10). Recent studies have shown that some EGF residues like Arg-41 and Leu-47 are highly conserved and important for high binding affinity to EGFR (11). Another study highlighted Tyr-13, Leu-15 and His-16 in EGF as essential for downstream activity of ErbB1 (12). These outcomes were based on structural analyses of ligands and experimental validation. While bioinformatic tools such as contact prediction (13) or molecular dynamics (14) can give a good overall picture of ligand-receptor interactions, the contribution of single ligand residues to the modularity of the system still remains unclear.

Ligand-induced selective activation of downstream pathway has been observed in G-protein coupled receptors, a phenomenon known as "biased signaling" $(15,16)$. A recent report hints that this mechanism might also take place in EGFR tyrosine kinase (17). Initially, the main contributor to the modularity of the system was thought to be the affinity of the ligands to the receptor. However, the discovery of the ligand EPGN, which induces a potent mitogen effect despite low binding affinity (18), and multiple cell-line studies (5) have changed this perspective. The standing theory is that different EGFR ligands induce different receptor dimer

91 stabilities, altering the phosphorylation dynamics; stable receptor dimers show transient activation, reaching the phosphorylation level required to initiate negative feedback signals 
93 faster than the unstable counterparts (19). Now, the open question is how these ligands induce

94 different dimer stabilities. Interactions between the interface of the ligand and EGFR have been

95 shown to influence the stability of the dimerized receptor (20), a factor that is related to ligand-

96 specific signaling bias $(19,21)$. It is known that the EGFR dimer can be observed in a

97 symmetrical, "flush" conformation or an asymmetrical, "staggered" conformation (22)

98 depending on the presence/absence of the membrane, glycosylation, and the number of bound

99 ligands (23). Whether and how this plays a role in biased signaling is still unknown.

100

101 Here we show how single amino acid substitutions alter biased signaling in the EGF-EGFR

102 ligand-receptor system. We developed a new bioinformatic tool to analyze the co-evolution of

103 the ligand-receptor pair and identify candidate function-altering mutations. The identified

104 mutants induced an altered phosphorylation dynamics and different cellular phenotype. This is

105 the first study to explain differences in biased signaling of EGFR using single-residue EGF

106 mutants. Furthermore, our co-evolutionary analysis can be applied readily to other ligand-

107 receptor interactions. 

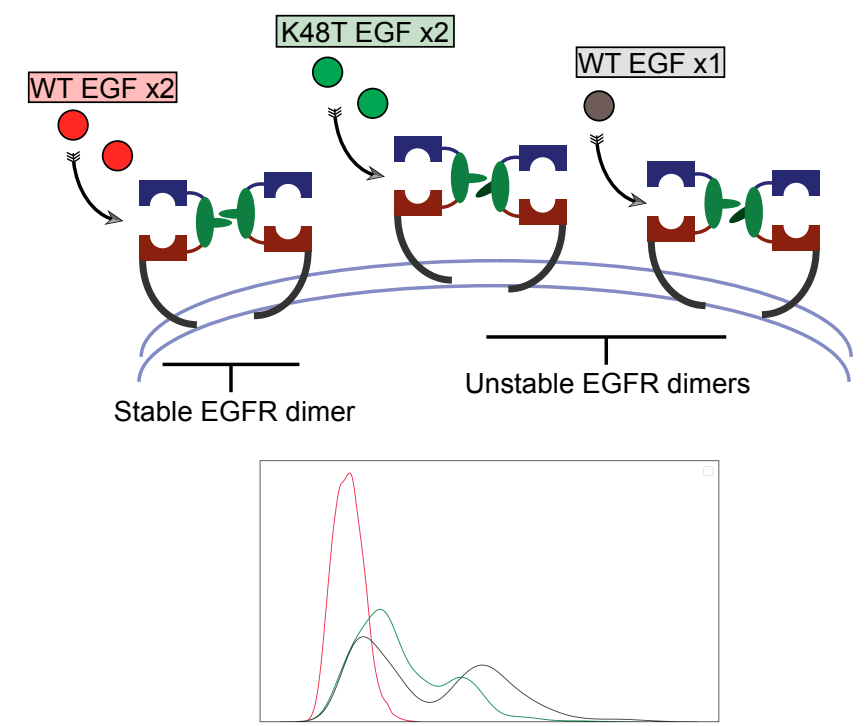

\section{Dimer dynamics}

110 Figure 1. Study rationale. EGF K48T mutant was identified using an ad hoc methodology

111 that combines conservation and coevolution measures. Unlike WT EGF, two K48T ligands

112 induce an unstable conformation of the receptor dimer, similarly to a dimer with a single WT

113 EGF. Treating A431 cells with either WT EGF or K48T mutant EGF resulted in a different

114 response both in the phosphorylation dynamics and the cell proliferation phenotype.

116 Results

\section{DIRpred}

119 Firstly, we developed a method for predicting residues that are likely to be responsible for

120 functional differences induced by paralogous ligands of a given receptor, which we called

121 DIRpred (Divergence Inducing Residues prediction). Our approach combines residue-specific

122 conservation measures to identify positions that are conserved among orthologs while

123 diverging among paralogs. The DIRpred analysis is based on the assumption that conservation

124 of a residue in orthologs of a specific ligand shows whether a residue is important for either 
125 structural or functional reasons, while conservation of a residue among paralogous ligands

126 denotes the importance of a residue for receptor binding (the main shared property of all

127 ligands). Thus, residues that are highly conserved in orthologs but not paralogs of a ligand are

128 likely related to the ligand's specific function. Unlike other existing methods for prediction of

129 specificity determining residues (a review can be found in (24)), we included inter-protein

130 coevolution measures to narrow down those residues that are responsible for a specific function.

131 The DIRpred score is calculated as the sum of the four components (I: orthologs conservation,

132 II: complement of the paralog's conservation, III: ligand-receptor co-evolution, IV:

133 complement of ligand internal co-evolution). Optionally, the analysis can be conducted using

134 a structural alignment of the paralogs instead of the sequence alignment.

\section{DIRpred analysis of EGF}

137 We applied the DIRpred algorithm to predict single amino acid substitutions in EGF that would

138 alter its function (Figure 2). Since the analysis requires prior knowledge of paralogs and

139 orthologs of the target gene, we first performed a phylogenetic analysis to confirm that the

140 reported paralogous ligands of EGF are monophyletic (Supplementary figure 1). The DIRpred

141 analysis highlighted residue Asn-32, Asp-46, Lys-48 and Trp-50 as potential candidates for

142 paralog functional divergence. Asn-32 and Lys-48 show a very small conservation in both

143 sequence and structure alignments resulting in a high partial score (II). Asp-46 has a relatively

144 high coevolution with the receptor (III), while Trp-50 has high scores overall (Figure 2 -

145 figure Supplement 1). We chose to introduce orthologous substitutions at these four positions,

146 as orthologous have been previously observed as enriched in function (see Materials and

147 Metods) (25). The four designed EGF mutants with a single amino-acid substitution that were

148 selected for functional characterization were N32R, D46T, K48T and W50Y. 
A
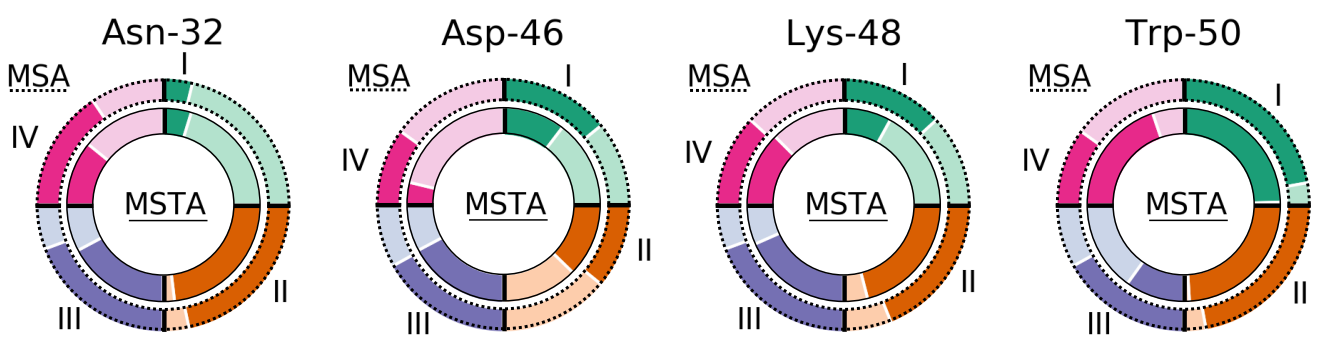

B

C
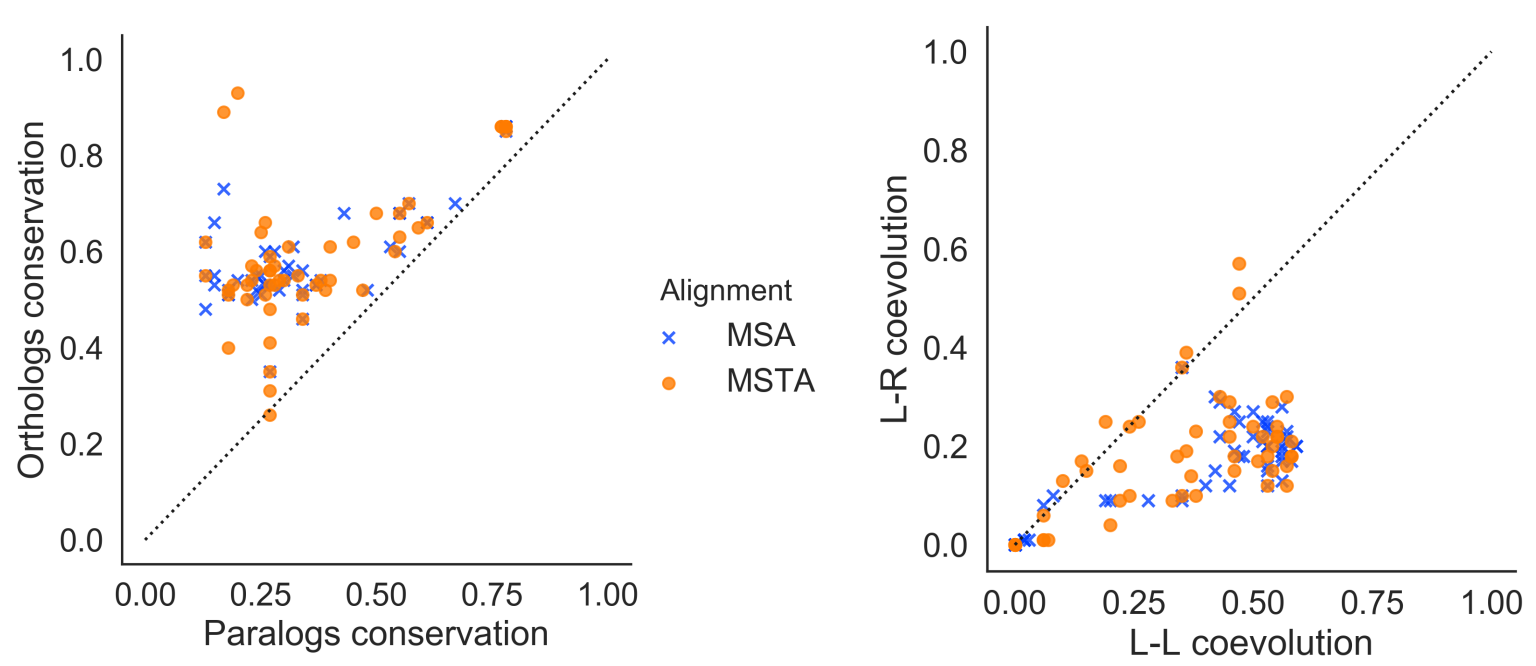

150 Figure 2. DIRpred analysis of EGF. (A) The relative ranking score of the four mutants. The outer circle represents paralogs MSA-based scores, while the inner circle represents paralogs MSTA-based scores. The darker color indicates a higher ranking in the EGF sites-based scoring. (B) Cross-conservation plot. The plot is obtained by crossing the two conservation scores.

154 Interestingly, no point lies in the bottom right half of the plot (high paralogs conservation and

155 low orthologs conservation), suggesting that paralogs and orthologs conservation are not independent. This observation points out that there is no organism-specific adaptation shared

157 by all ligands at the protein sequence level. (C) Cross-co-evolution score. The plot is obtained

158 by crossing the two co-evolution scores, the ligand-receptor (L-R) co-evolution score (III) on

159 the y axis and the ligand-ligand (L-L) co-evolution score (IV) on the $\mathrm{x}$ axis. 
I) Orthologs conservation

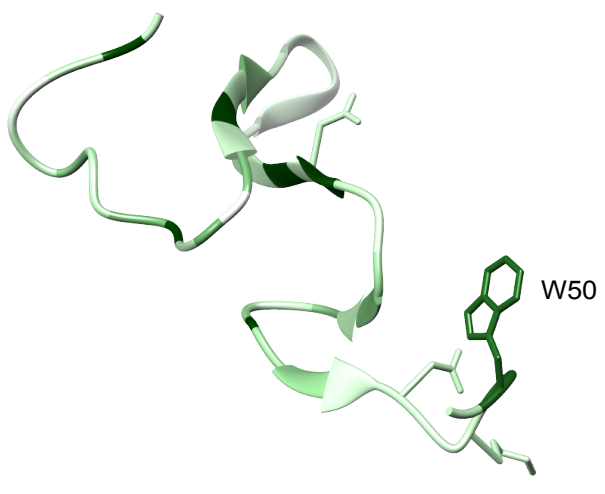

III ) Ligand-receptor Co-evolution

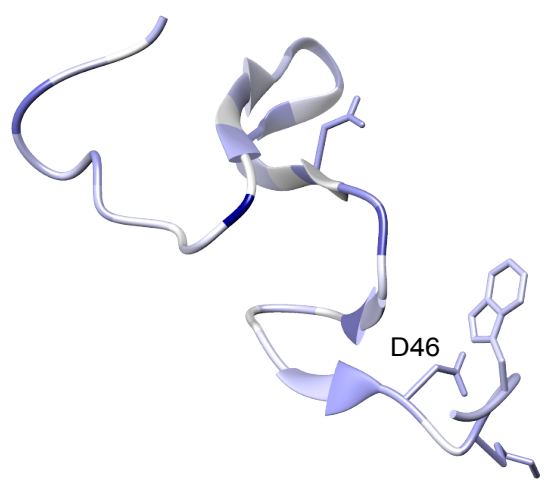

II ) Paralogs MSA/MSTA conservation (neg)

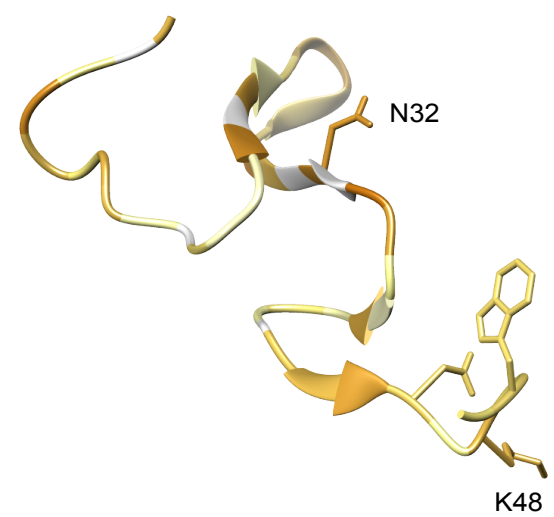

IV ) Ligands internal Co-evolution (neg)

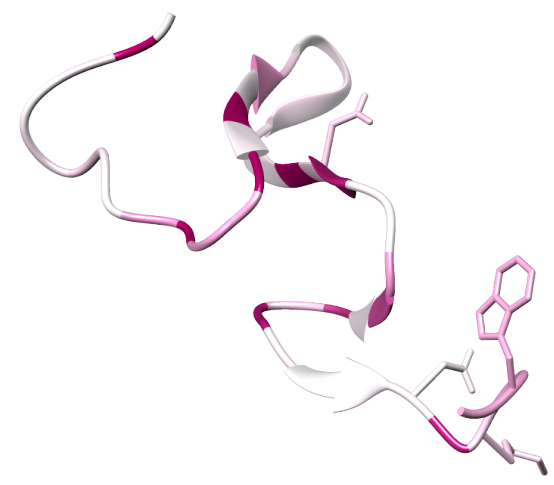

160

161 Figure 2-figure Supplement 1. DIRpred score. Structure of EGF (from PDB: 1IVO)

162 colored by the four individual scores. The four sites chosen for mutation are shown in stick

163 representation. 
A
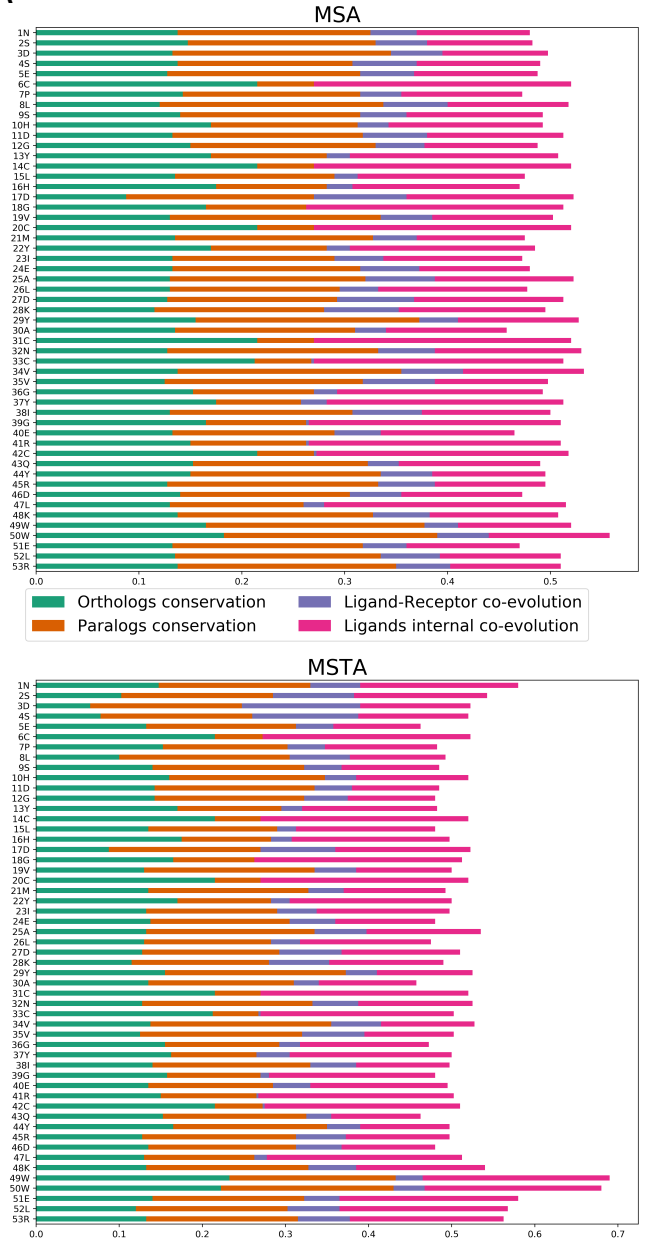

\section{B}
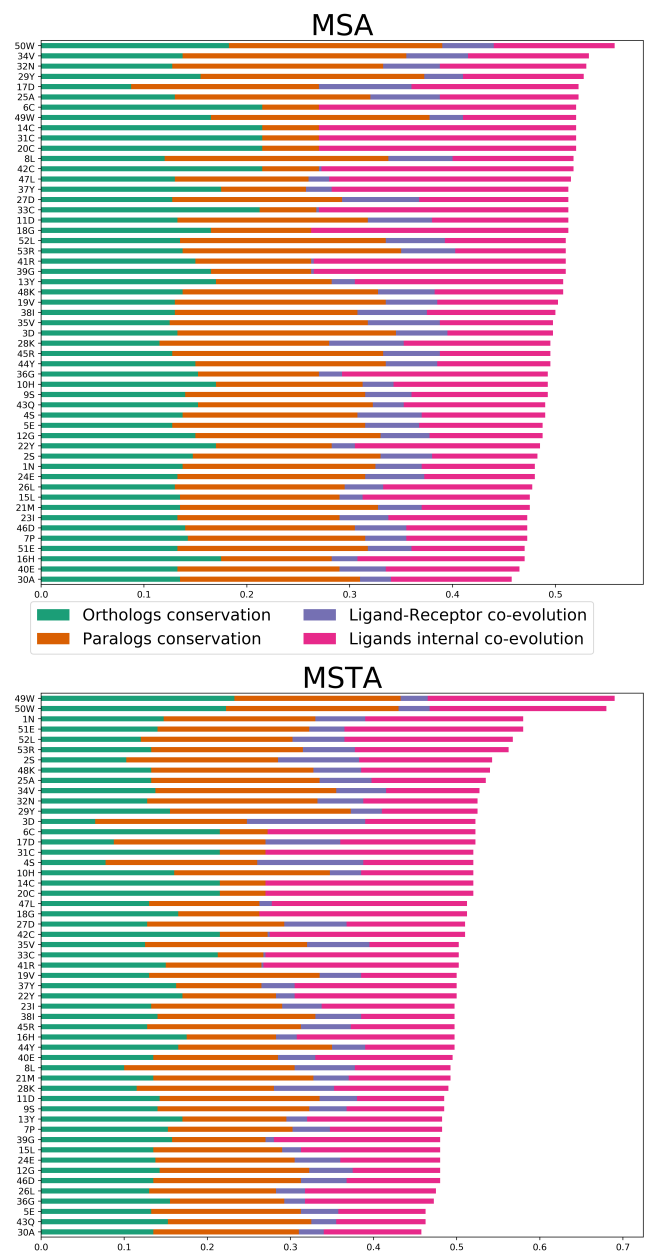

165 Figure 2-figure Supplement 2. All sites score. This figure shows the four scores for each

166 site of EGF. On the top, the paralogs MSA based scores, while on bottom, scores are based on

167 the paralogs MSTA. In (A) the scores are in order of sequence. In (B), the scores are sorted by

168 highest sum first. 


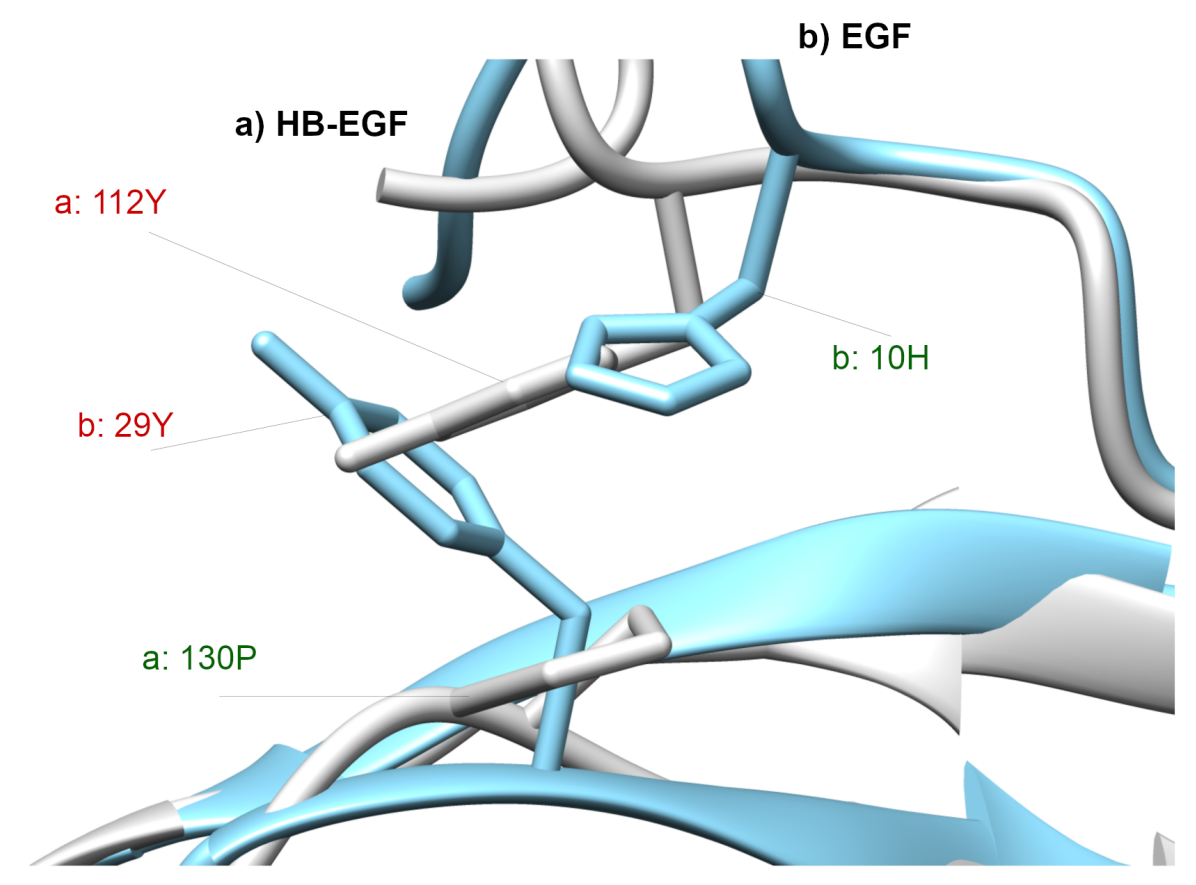

170 Figure 2-figure Supplement 3. Highlight on the EGF-specific residue swapping problem.

171 EGF is colored in teal, while HB-EGF is colored in white. The tyrosine in EGF position 29

172 (29Y) is not found on the corresponding position of HB-EGF. Instead, a proline is found (130P).

173 Though, the interaction between the two residue is probably maintained because, in place of

174 the histidine $10(10 \mathrm{H})$ of EGF, HB-EGF shows a tyrosine (112Y). When this type of residue swapping is ligand-specific, the lowering of the conservation measures over-estimates the real change happening in that position.

\section{Biochemical properties of the EGF mutants}

179 To determine the functional effects of these amino acid substitutions, we first performed $\mathrm{in}$ -

180 vitro analysis. Initially, Circular Dichroism (CD) spectroscopy was used to confirm that the

181 secondary structure of the mutants was maintained (Figure 3-figure Supplement 1). Then, the

$182 \mathrm{~K}_{\mathrm{d}}$ of each mutant for EGFR was determined by Isothermal Titration Calorimetry (ITC). ITC

183 measurements of the binding of all mutants to the ECD of EGFR exhibited similar $\mathrm{K}_{\mathrm{d}}$ values 

in-vitro. Thus, the mutations do not appear to affect the receptor through a change in affinity.

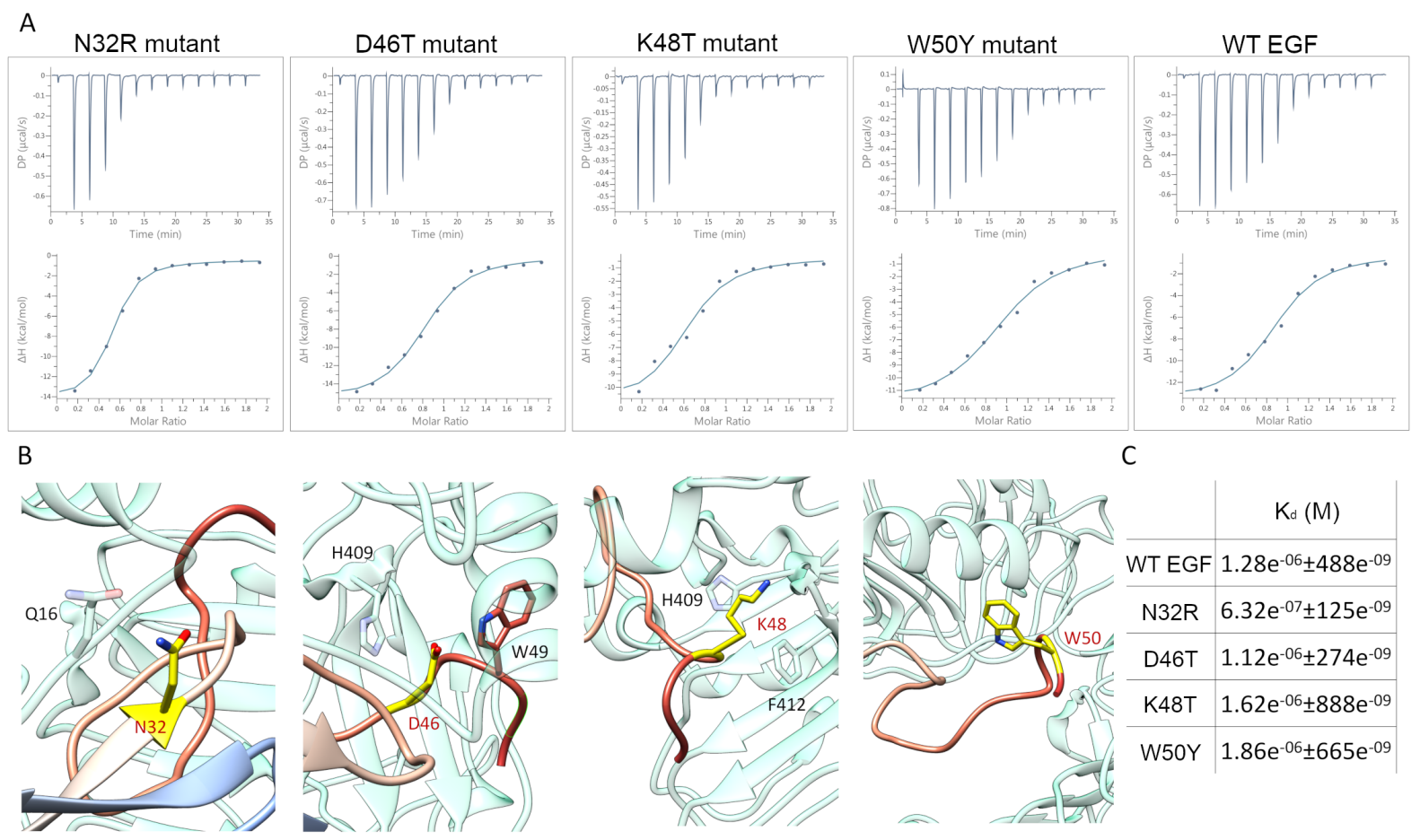

188 Figure 3. ITC measurements of EGF mutants and the EGFR receptor. (A) ITC analysis

189 of WT EGF ligand and mutants N32R, D46T, K48T, and W50Y binding to the ECD of the

190 EGFR receptor at $25^{\circ} \mathrm{C}$. Measurements were taken by adding WT EGF at $200 \mu \mathrm{M}$ to the ECD

191 of EGFR at $20 \mu \mathrm{M}$. (B) Four zoom-in of X-Ray structure of the extracellular domain (ECD) of

192 EGFR bound to EGF (PDB 1IVO). In cyan cartoon the ECD of EGFR. Each zoom-in focuses

193 on the mutated residue. Highlighted in yellow stick side chain of the mutated residues and in

194 cyan stick side chain of the residue of ECD in proximity ( $<5 \AA$ with the mutated residue. (C)

$195 \mathrm{~K}_{\mathrm{d}}$ calculated from the ITC measurements using the program Affinimeter KinITC Kintecs

196 Software. 


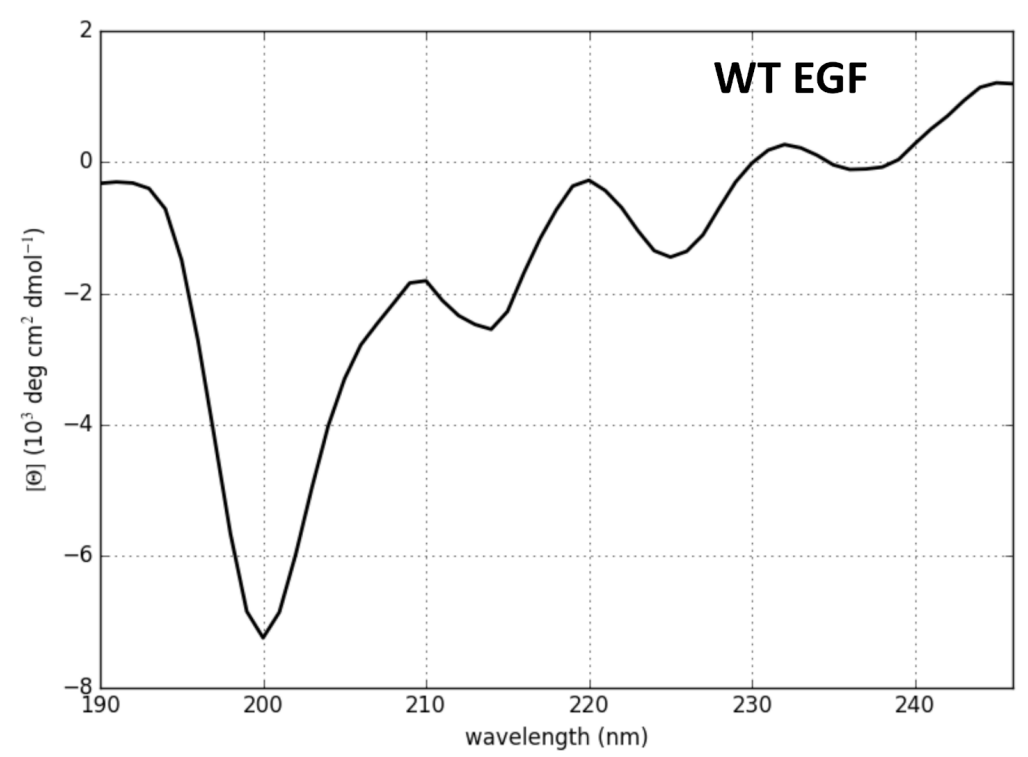

\begin{tabular}{c|c|} 
& $\% \beta$-sheet \\
\hline WT EGF & 0.44 \\
\hline N32R & 0.41 \\
\hline D46T & 0.47 \\
\hline K48T & 0.54 \\
\hline W50Y & 0.52
\end{tabular}

198 Figure 3-figure Supplement 1. The validation of ordered structure of WT EGF and variants. $0.2 \mathrm{mg} / \mathrm{mL}$ of the sample was added to a $10 \mu \mathrm{m}$ cuvette and analyzed by circular dichroism spectroscopy. All samples showed the presence of a comparable amount of $\beta$-sheet. The CD spectrum of EGF was similar to previously published results (26). The plot and analysis was made using CAPITO web tool (27).

\section{Biological effects of the EGF mutants}

Next, we sampled the effect of the EGF single mutants on EGFR expression and phosphorylation in A431 cells. A431 is a human epidermoid carcinoma cell line that overexpresses EGFR, thus making it easier to observe differences at the receptor level. Treating this cell line with very low (pM) concentrations of EGF has a small effect on proliferation, while high concentration treatments $(>10 \mathrm{nM})$ induces apoptosis $(28)$. We measured the amount of total and phosphorylated EGFR protein at multiple timesteps by Western Blot after

211 treating with saturating concentrations (100 nM) of WT or mutant ligands (Figure 4). 
212 Remarkably, K48T and W50Y showed a greater increase in EGFR phosphorylation 1h after

213 treatment than WT EGF (Figure 4-figure Supplement 1). Treatment with the mutant ligands,

214 but not the WT ligand, also caused a reduction in the amount of EGFR $1 \mathrm{~h}$ after treatment. At

215 later time points, the effects converge back to the same range of the No Ligand (NL) sample

216 (Figure 4-figure Supplement 2). Thus, while the EGF mutants showed comparable binding

217 affinities to the receptor, at least two of the mutants showed different phosphorylation

218 dynamics compared to the WT ligand. Furthermore, the observed differences in the total

219 amount of EGFR protein indicate a possible alteration in the membrane expression or recycling

220 of the receptor. 
A

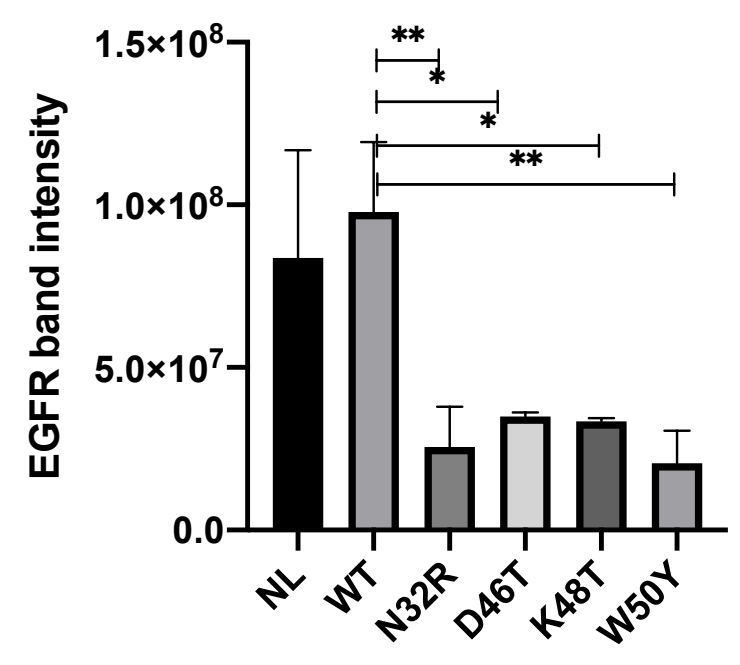

C

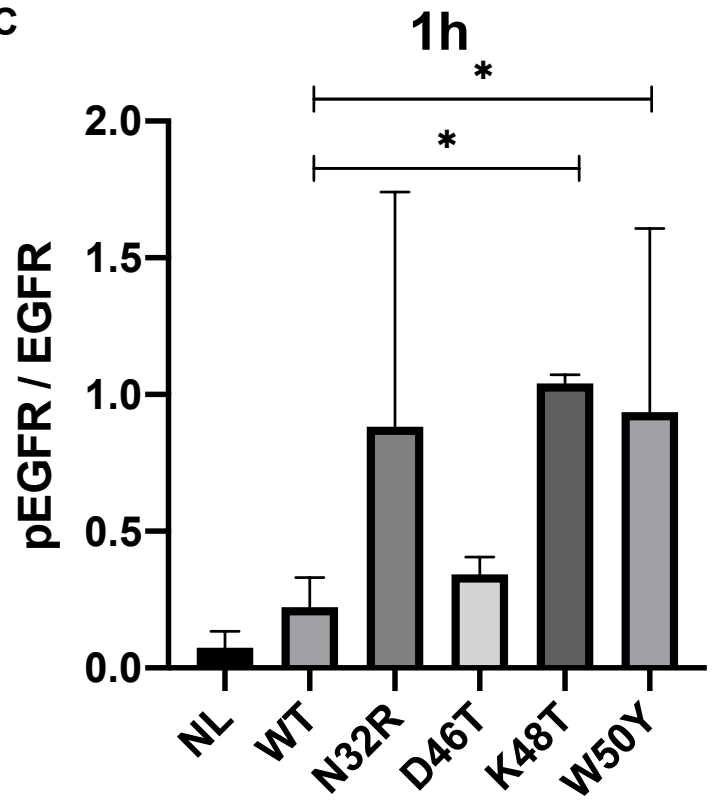

B

$6 h$

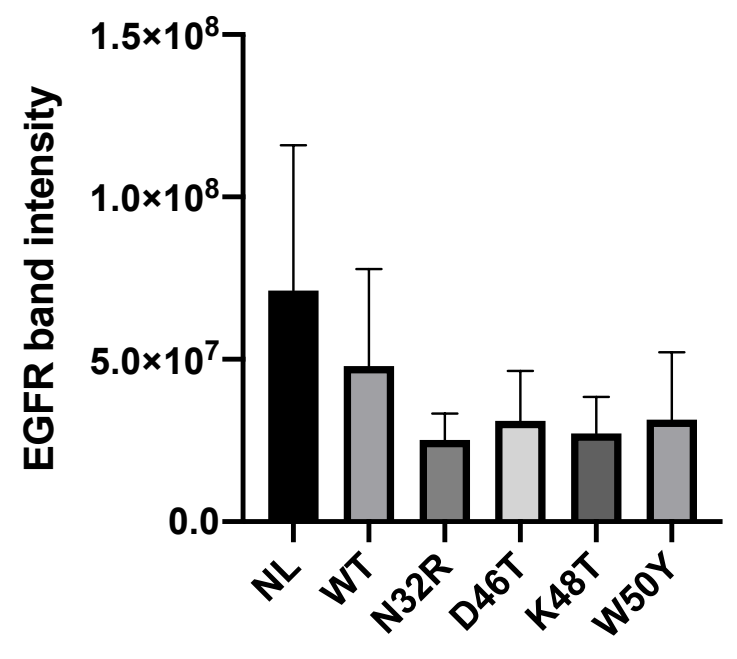

$6 h$

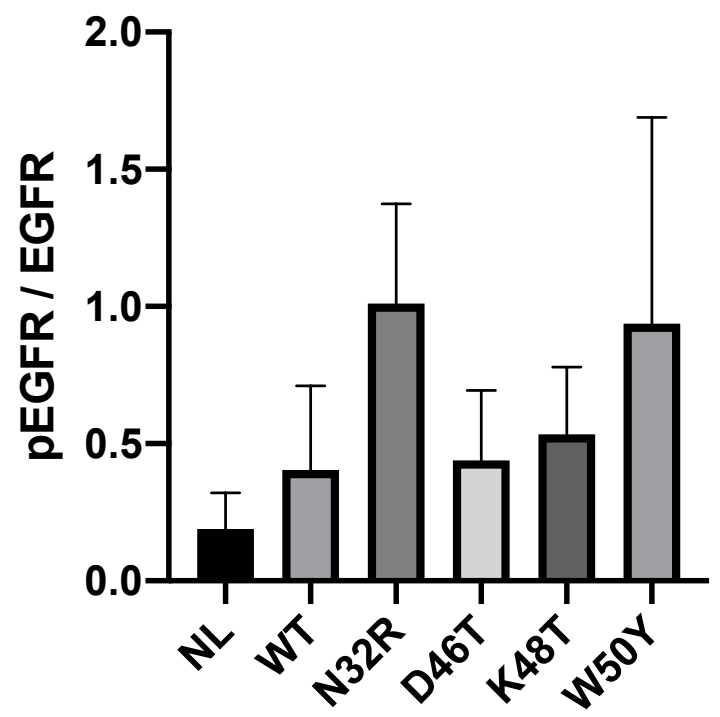

222 Figure 4. Effects of WT and single-mutant EGF on the receptor in A431 cells. (A-B)

223 Intensity of EGFR band after $1 \mathrm{~h}(\mathrm{~A})$ or $6 \mathrm{~h}(\mathrm{~B})$ treatment of saturating concentrations $(100 \mathrm{nM})$

224 of the different ligands. (C-D) Relative phosphorylation level, estimated as the ratio of pEGFR

225 (Tyr-1173) band intensity to EGFR band intensity, at $1 \mathrm{~h}(\mathrm{C})$ or $6 \mathrm{~h}$ (D). All intensities were 226 normalized using the respective alpha-tubulin bands. The bars represent mean \pm s.d. $(n=2$, 
227 technical replicates). Band intensity estimates were calculated using BIO-RAD ImageLab

228 software (BioRad). Plots and statistics were performed using PRISM software (GraphPad).

A
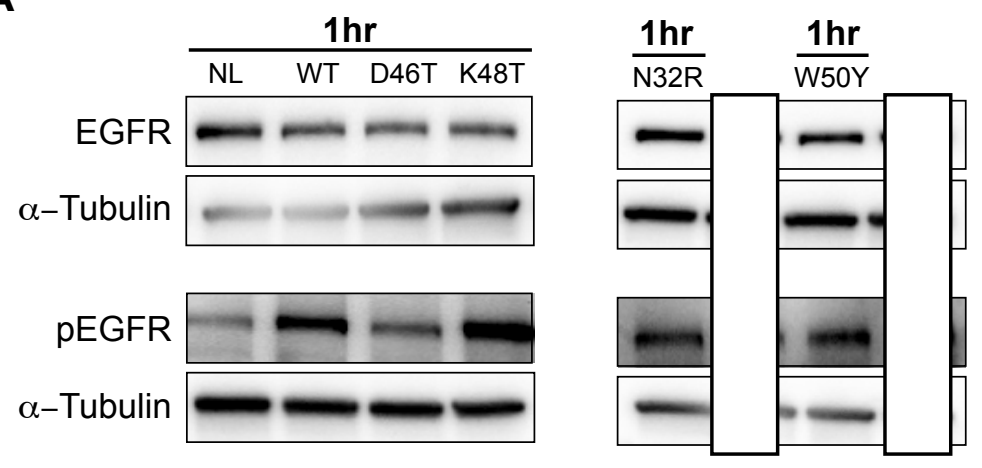

B
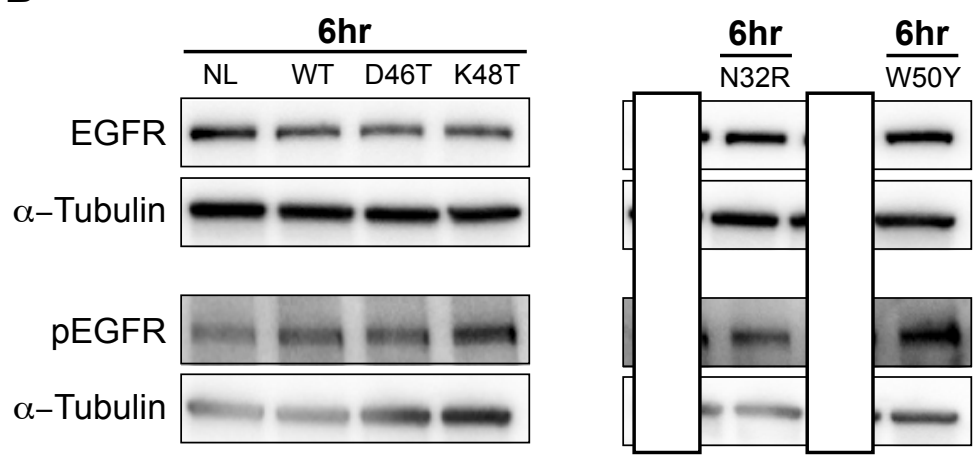

230 Figure 4-figure Supplement 1.1h and $6 \mathrm{~h}$ post-treatment Western Blots. Image of one

231 duplicate of A431 treated cells western blot for $1 \mathrm{~h}(\mathrm{~A})$ and $6 \mathrm{~h}(\mathrm{~B})$. The membrane containing

232 EGFR and tubulin was separated after the transfer. N32R and W50Y gel is the same in both

233 rows, but its lanes have been obscured to facilitate comparison at the same time steps with

234 other samples. 
A

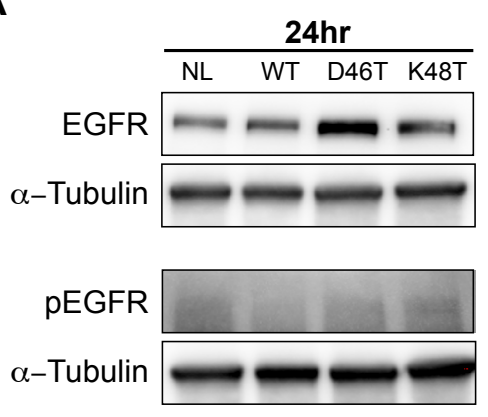

B

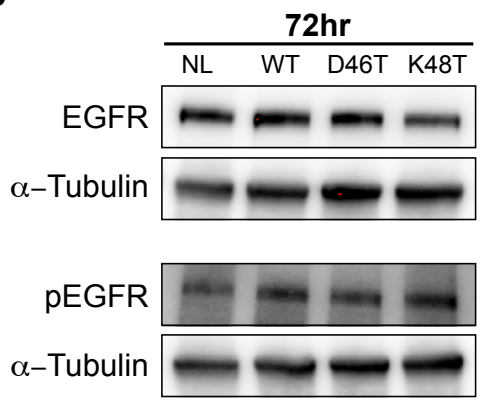

C

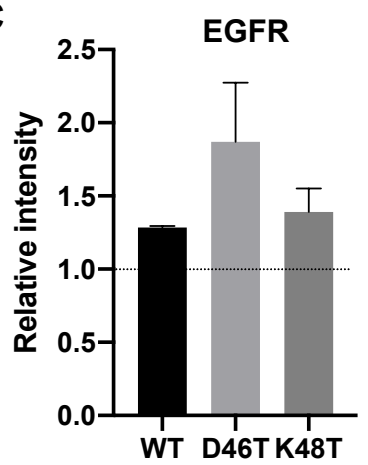

D

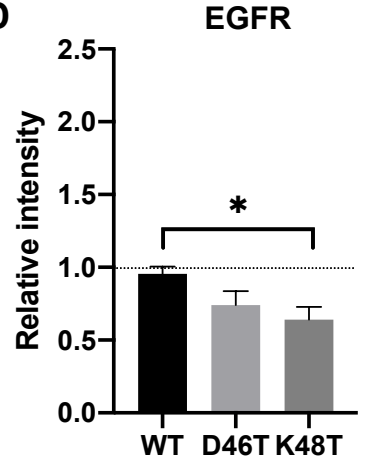

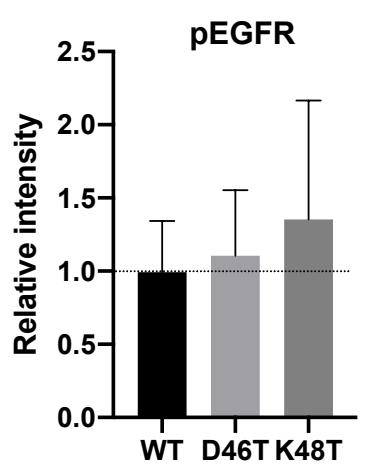

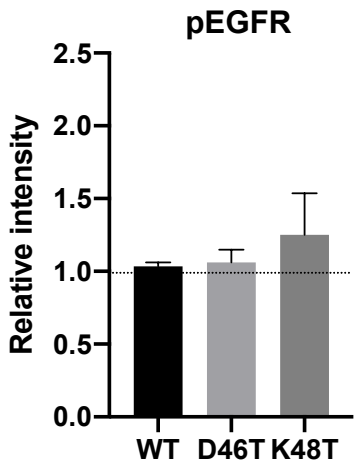

$\mathbf{E}$
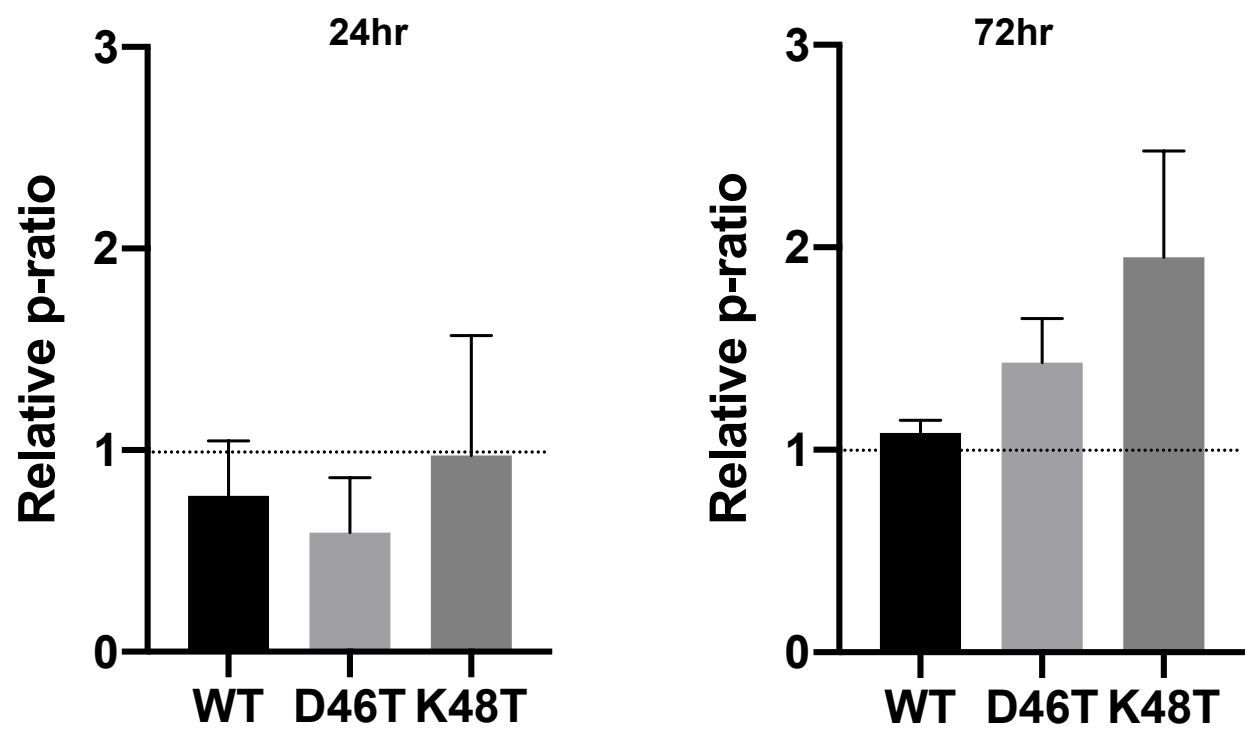

236 Figure 4-figure Supplement 2. Effects on the receptor at $24 \mathrm{~h}$ and $\mathbf{7 2 h}$. (A) Western blot of A431 cells lysate after treatment with No Ligand (NL), WT EGF, D46T or K48T mutants $24 \mathrm{~h}$ after treatment. (B) Image of the same experiment collected at $72 \mathrm{~h}$ after treatment. (C) EGFR and pEGFR (Tyr-1173) band intensities, relative to the NL sample at $24 \mathrm{~h}$ and (D) at $72 \mathrm{~h}$

240 after treatment. In (E) and (F), the relative phosphorylation ratio compared to NL respectively at $24 \mathrm{~h}$ and $72 \mathrm{~h}$. A value of 1.0 represent the same phosphorylation ratio as observed in NL 
242 lanes. All intensities were normalized using the respective alpha-tubulin bands. The plots were obtained from duplicate of technical repetitions. Band intensity estimates were calculated using

244 BIO-RAD ImageLab (BioRad). Plots and statistics were performed using PRISM software 245 (GraphPad).

247 In order to observe the effects of the mutants on the cellular phenotype, we performed a cell 248 growth assay using an IncuCyte ${ }^{\circledR}$ live-cell analysis system. After one day of initial incubation, 249 we subjected Bj5-t $\alpha$ and A431 cells to 1,10 or $100 \mathrm{nM}$ concentration of growth factors. 250 Treating Bj5-t $\alpha$ fibroblasts with $100 \mathrm{nM}$ WT EGF resulted in a reduction in the proliferation 251 rate compared to the control. The reduction in proliferation was accompanied by a change in cellular morphology that could be a signal of differentiation (Supplementary data 1). However, treatment with any of the mutants decreased the ability of EGF to suppress growth (Figure 5A). The D46T and K48T mutants differed most from the WT (Figure 5-figure Supplement 1). We observed a similar trend in the Albino Swiss mouse 3T3 cell line (Figure 5-figure Supplement 2). In both cell lines, the effects are concentration dependent (Supplementary figure 3). Next, we further tested the mutants in an apoptosis assay using the A431 cell lines. This cell line is known to exhibit apoptosis and reduced growth rate when treated with EGF peptide (29). $48 \mathrm{~h}$ after treatment with $100 \mathrm{nM}$ WT or mutant EGF, the WT showed the highest

260 decrease in cell proliferation, while the four mutants had an intermediate effect. Cells treated with the W50T mutant had the closest proliferation level to the WT (Figure 5B). In WT EGF cells, we observed evidence of apoptosis in globular processes. Meanwhile, K48T treated cells displayed signs of cellular differentiation (Figure 5C). Significantly, these results show that

264 single amino acid changes in the EGF ligand display differential effects on the EGFR transduction mechanism. 
A

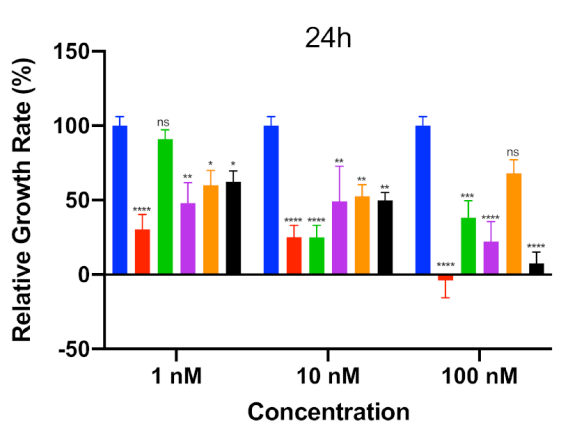

B

A431 Human Cancer Cell Growth

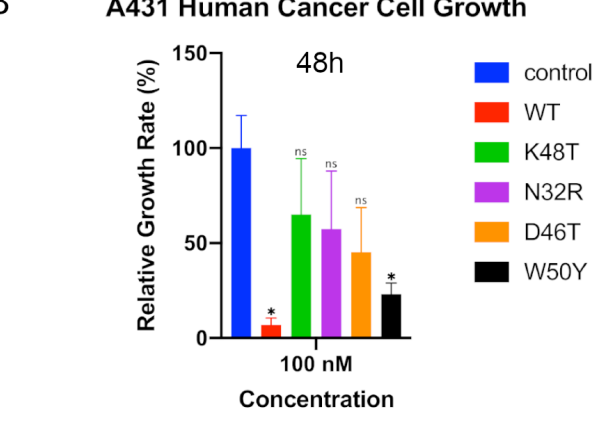

Growth

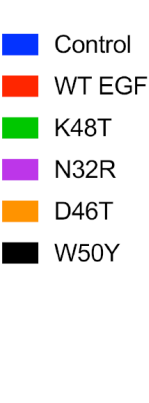

C A431 Human Cancer Cells

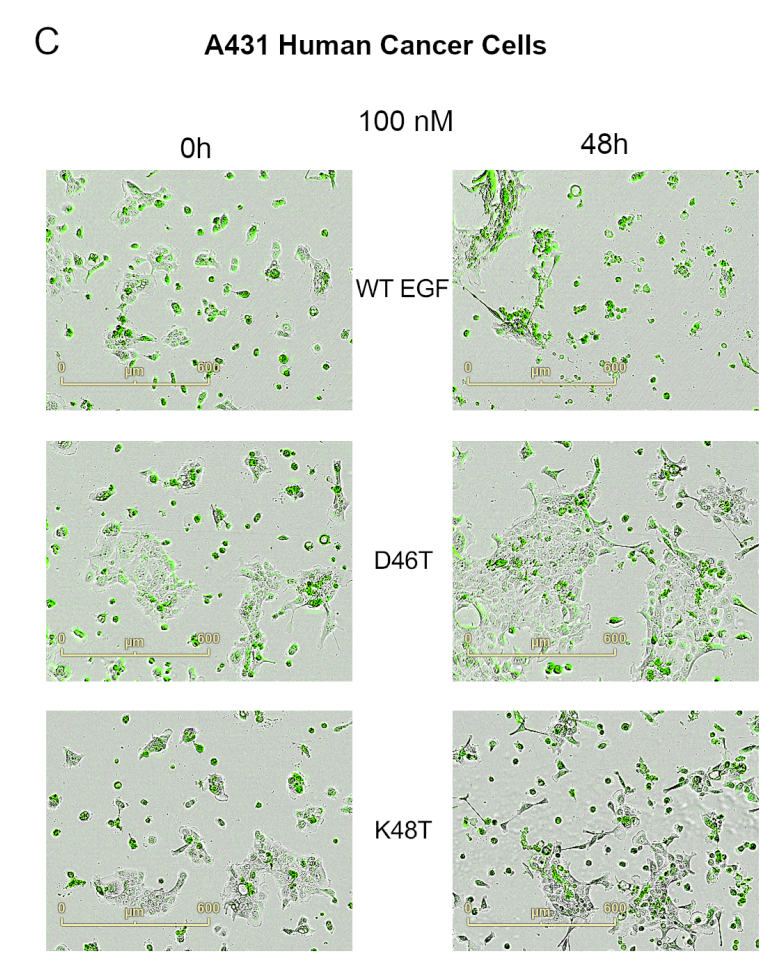

267 Figure 5. Growth assays of cells treated with EGF variants. (A) Effect of different

268 concentrations of EGF variants on proliferation of the human normal fibroblast Bj5-t $\alpha$ cell line.

269 Data represent the growth rate percentage calculated from the confluence of cells relative to

270 the control (mean \pm standard deviation) for each concentration of EGF. Percent confluence was

271 estimated $24 \mathrm{~h}$ after the treatment (two replicates per treatment). (B) Effect of EGF mutants on

272 the growth of A431 cells. EGF-induced apoptosis is measured as the reduction in cell

273 proliferation compared to the control. (C) Comparison of A431 cell growth after treatment with

$274100 \mathrm{nM}$ WT EGF and EGF variants D46T and K48T. Cells were labeled with fluorescent

275 Annexin V Green Reagent. Plates were pre-warmed prior to data acquisition to avoid

276 condensation and expansion of the plate, which affect autofocus. Images were captured every

$2772 \mathrm{~h}$ (4x magnification) for 3 days in the IncuCyte system. 


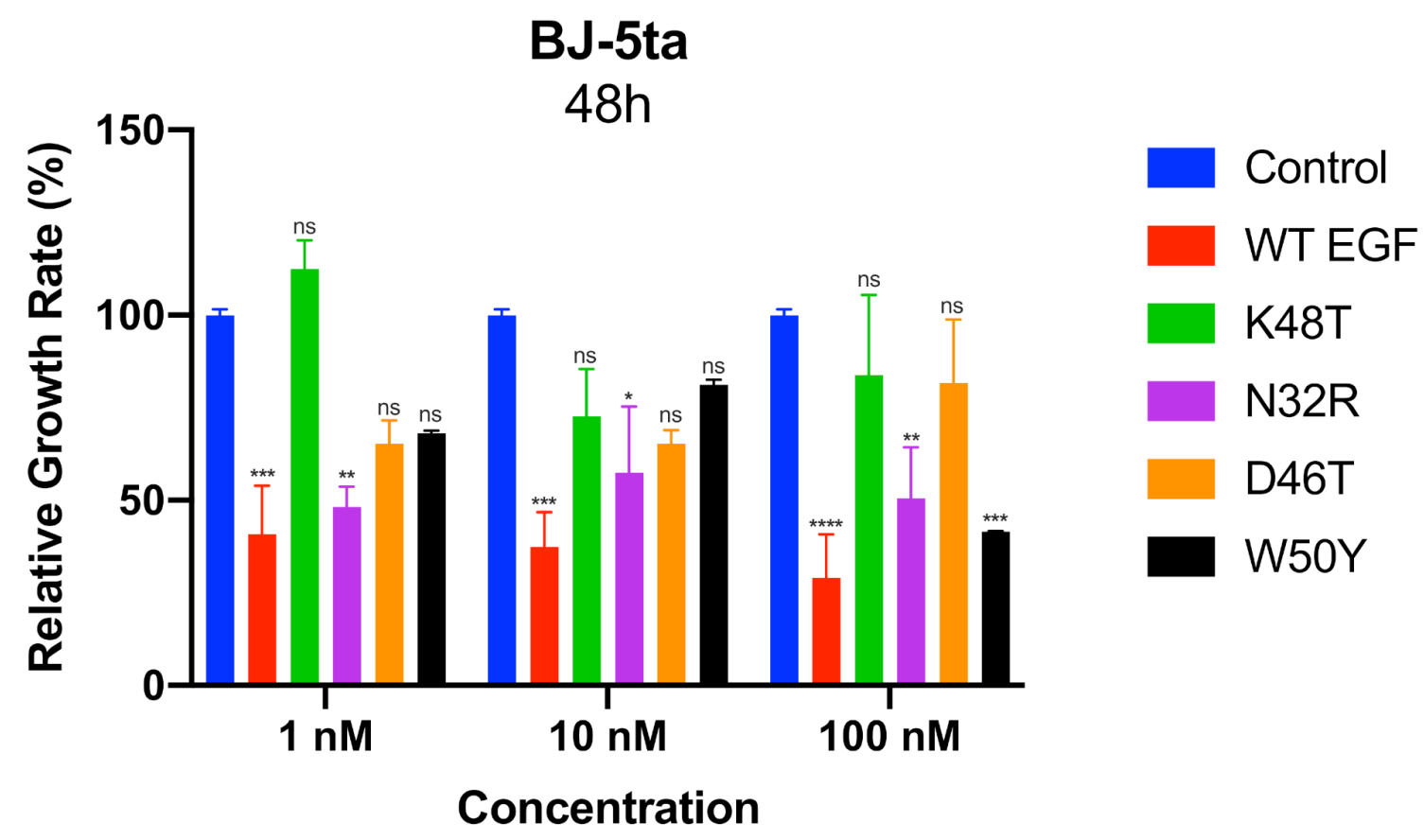

279 Figure 5-figure Supplement 1. Effect of 1nM, 10nM and 100nM concentrations of EGF

280 variants on the proliferation of the human normal fibroblast $\mathbf{B j 5}$-t $\alpha$ cell line. The data

281 represent growth as a percentage of the control based on the confluence of cells (mean \pm

282 standard deviation) for each concentration of EGF. The percentage confluence was estimated

$28348 \mathrm{~h}$ after the treatment (two replicates per treatment). 


\section{Albino Swiss 3T3 Mouse Normal Fibroblast Cells}

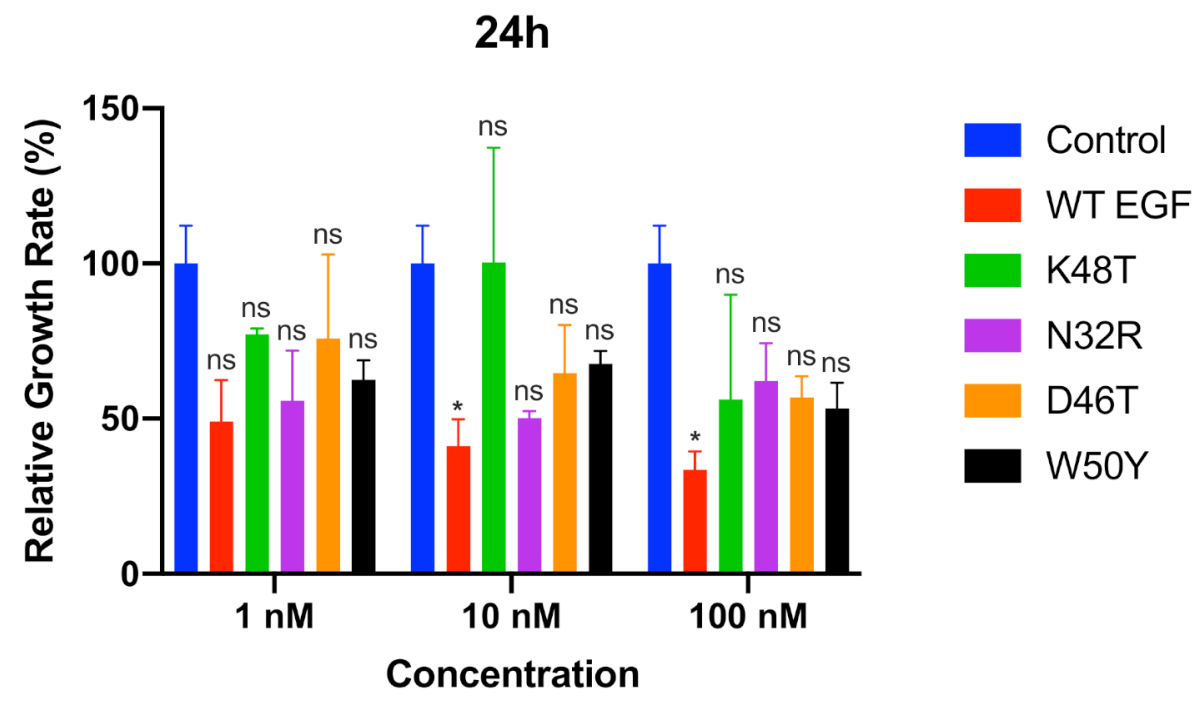

$48 h$

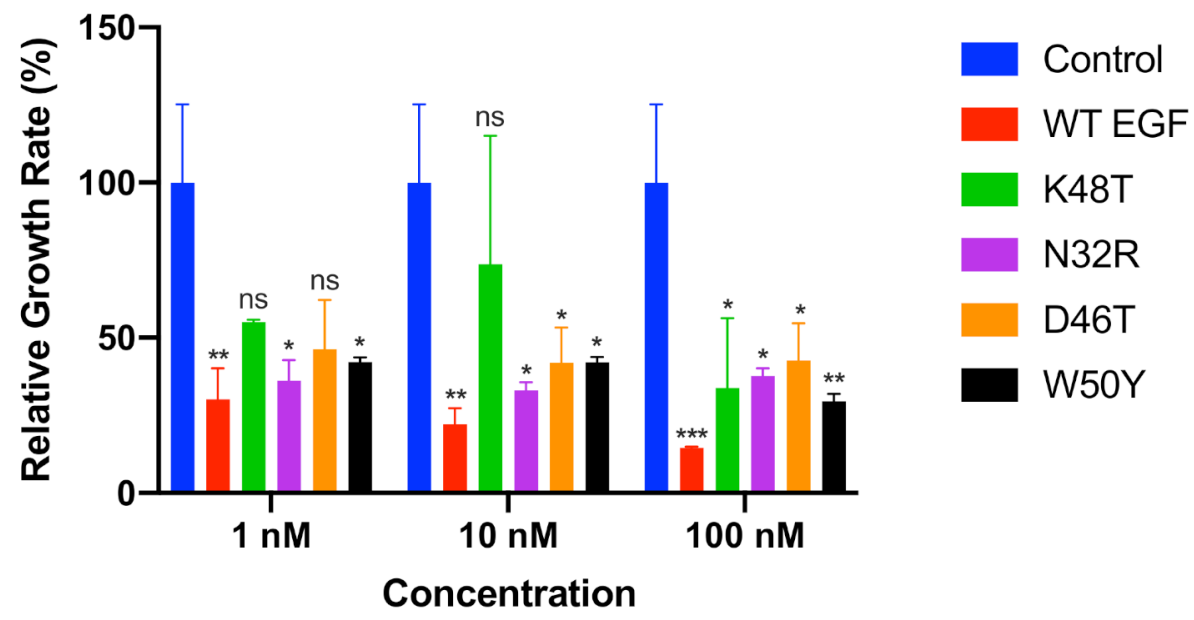

285 Figure 5-figure Supplement 2. Effect of 1nM, 10nM and 100nM concentrations of EGF

286 variants on the proliferation of the mouse normal fibroblast Albino Swiss 3 T3 cell line.

287 The data represent the relative growth percentage to control based on the confluence of cells

288 (mean \pm standard deviation) for each concentration of EGF. 


\section{Molecular dynamics}

292 To understand how EGF single mutants affect the receptor signaling transduction, we

293 performed full atomistic molecular dynamic (MD) simulations of the extracellular EGFR in

294 complex with WT or mutant ligands (100 ns each). We modeled the receptor starting from the

295 asymmetric, "unstable" conformation of 5WB7 (22). In this way, we expected to observe a fast

296 rearrangement for those simulations where a ligand more favorably induce a stable dimer. In

297 previous literature, an unstable conformation was observed when removing one of the EGF

298 ligands (23). We also performed one simulation of the EGFR dimer in complex with only one

299 WT ligand as a comparison (1ligEGF). The MD simulations quickly converged to a stable

300 RMSD (Figure 6A). For each simulation, we calculated the number of H-bonds between the

301 two receptor dimers, and between ligand and receptor. In the WT EGF simulation, the EGFR

302 dimer had an average of $15 \mathrm{H}$-bonds, higher than any other simulation (Figure 6-figure

303 Supplement 1A). However, the number of bonds between receptor and ligand was not altered

304 (Figure 6-figure Supplement 1B). In addition, during the course of our simulations we also

305 noted differences in the RMSF specifically located at the dimerization arm domain (Figure 6B).

306 The conformational space sampled by the dimerization arm of K48T simulation was much

307 wider than the WT simulation (Figure 6C). To analyze the temporal distribution of these

308 motion, we measured the distance between Pro-272 and Gly-288 of different EGFR monomers.

309 This distance was chosen because it was able to discriminate between the EGFR dimer in

310 complex with EREG (PDB ID 5WB7) and EGF (PDB ID 1IVO). In 5WB7, one of the two

311 distances is much bigger $(\sim 1.10 \mathrm{~nm}$ vs $0.4 \mathrm{~nm})$ compared to 1IVO (Figure 6-figure

312 Supplement 1C). In our simulations, we observe WT showing a sharp peak at $1 \mathrm{~nm}$, while the

313 mutants and 1ligEGF have a secondary peak at higher distances (Figure 6-figure Supplement

314 1D). Thus, the mutations in EGF appear to affect the stability of the EGFR dimer without

315 affecting the stability of the EGF-EGFR interaction. 
A

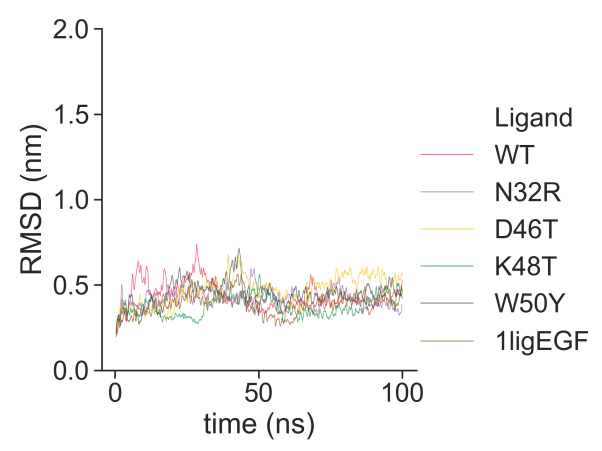

C

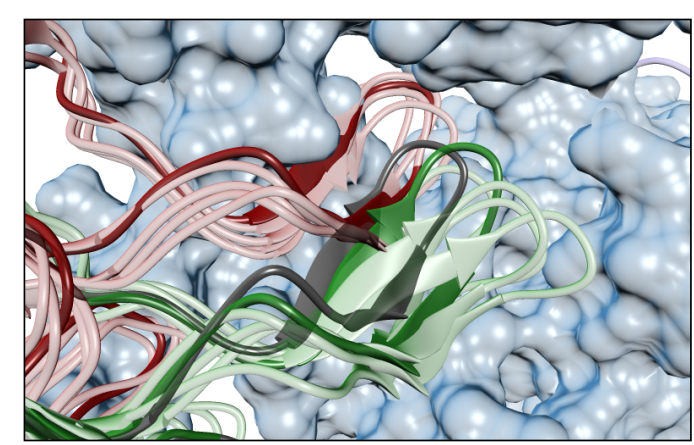

B
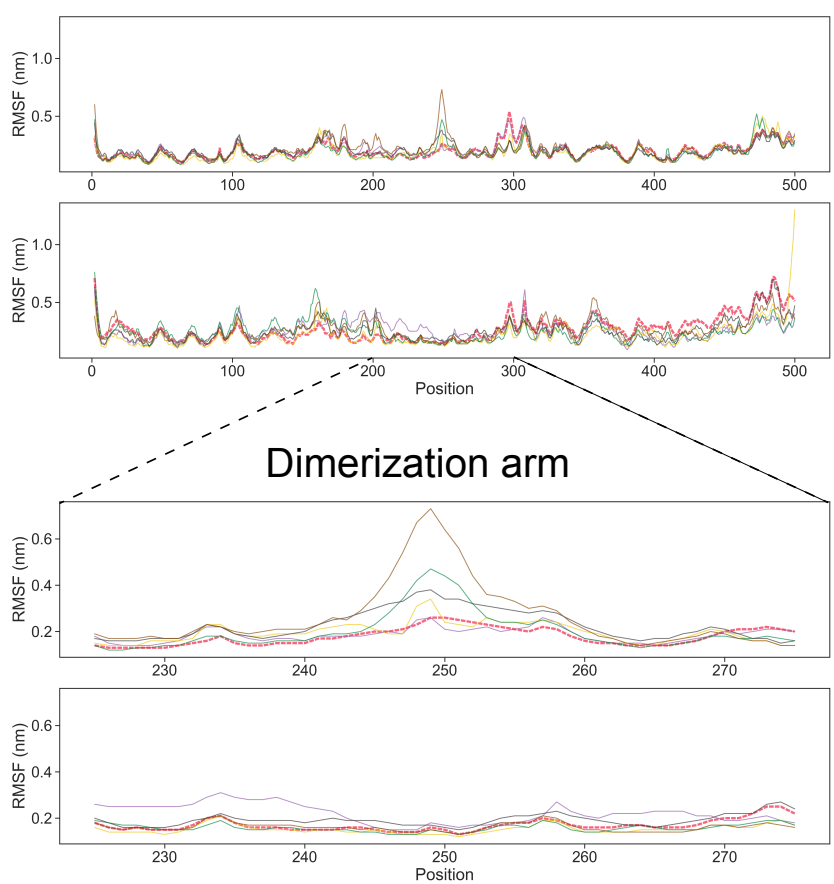

318 Figure 6. Molecular Dynamics of the EGFR extracellular domain. (A) Root Mean Square

319 Deviation from the initial structure. (B) Top, Root Mean Square Fluctuations of the $100 \mathrm{~ns}$

320 simulations. The WT level is marked in bold and dashed red line. Bottom, focus on the RMSF

321 of the dimerization arm domain. The two plots represent the two EGFR in the receptor dimer.

322 (C) Dynamics of the dimerization arm domain in WT (red) and K48T (green) simulations. A

323 structure every $20 \mathrm{~ns}$ of simulation was taken and aligned to 1IVO reference structure (blue

324 surface and black ribbon). The structure at $20 \mathrm{~ns}$ is represented in solid colors. K48T simulation

325 shows a more dynamic dimerization arm. 
A

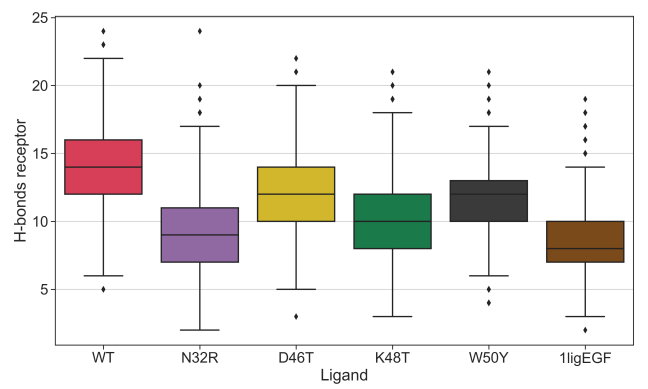

C

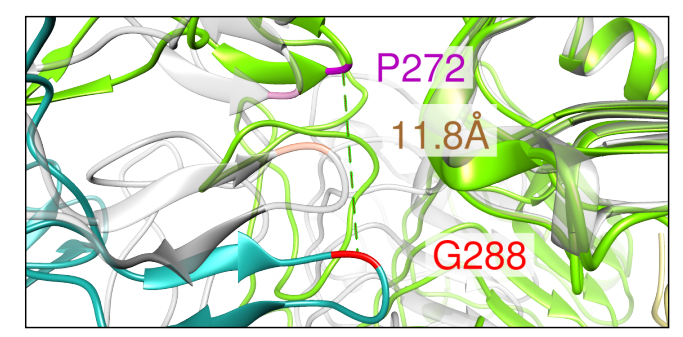

B

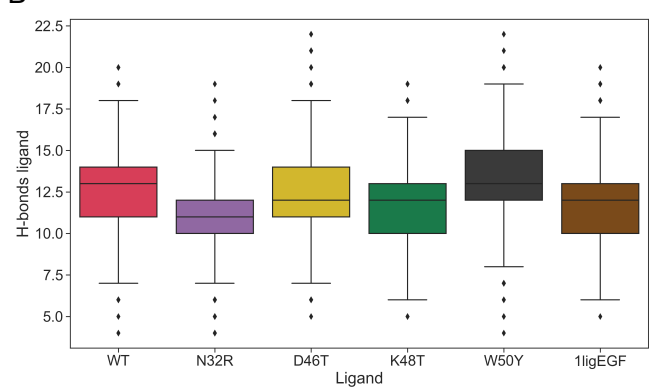

D

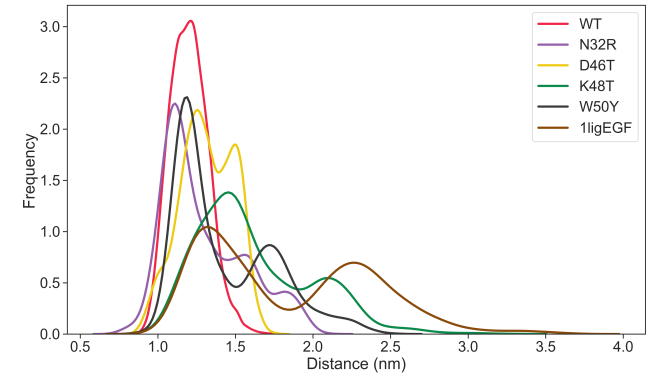

Figure 6-figure Supplement 1. RMSF and distance between Pro-272 and Gly-288. (A)

328 Number of $\mathrm{H}$-bonds formed between the two receptors during the $100 \mathrm{~ns}$ simulation time. (B)

329 Number of $\mathrm{H}$-bonds formed between the receptor that shows a dynamic dimerization arm and

330 the corresponding ligand during the $100 \mathrm{~ns}$ simulation time. (C) Distance used to investigate

331 the fluctuations of the dimerization domain highlighted on the structure. Pro 272 (purple) and

332 Gly 288 (red) are highlighted both on 5WB7 (green and teal) and in 1IVO (grey). The distance

333 between the alpha carbons of the 5WB7 couple is shown in brown and is notably longer than

334 1IVO. (D) Distribution of the distance between Pro272 and Gly 288 in all the simulations. WT

335 simulation shows a single peak at $\sim 1 \mathrm{~nm}$, while in all other ligands the distance has two peaks.

337 Discussion

338 The prediction of functional residues is a well-developed field (30), where conservation

339 measures are considered a key factor to rely on. Tools such as ConSurf (31) and the ET-like

340 methods (32) are able to identify slowly evolving positions that are involved in folding,

341 interaction, or catalytic activity (30). However, the specific reason why a residue is conserved

342 often remains unclear. In this work, we show a new method to identify residues that affects 
343 specific functions in a system of interest. Our approach combines a conservation score

344 calculated from the structural alignment of paralogs and among orthologs, with an intra- and

345 inter-molecular co-evolution score with previously known interactors. Conservation and

346 coevolution give a complementary signal, thus improving the overall predictive performance

347 (33). The coevolution score was introduced to DIRpred to highlight residues that are directly

348 responsible of an interaction with the receptor, at the expenses of those that interact

349 intramolecularly within the ligand. While the first part efficiently identifies Asn-32 and Lys-

35048 as putative interactors, the second part does not properly give a penalty to residue Tyr-29.

351 The interaction of this position with His-10 is also conserved in EGF but with reversed

352 positioning (Figure 2 - figure supplement 3), resulting in a low paralog conservation (then high

353 contribution to DIRpred score). While it is still possible to optimize the co-evolution scoring

354 function, integrating conservation and coevolution measures is a promising way to recall

355 information of specific functions involving protein-protein interactions.

357 Three out of the four positions were highlighted by previous reports, however none of them were individually mutated. The tryptophan in position 50 is a strong outlier in our bioinformatics analysis, along with Trp-49 (Figure 2). Their score is high even when using 360 conservation measures that do not take amino acid type change into account (data not shown).

361 However, Trp-50 was a better candidate for testing because of its outward facing position, and 362 since Trp-49 is involved in buried protein contacts (34). Trp-49 and Trp-50 could be responsible in facilitating the interaction of EGF with the membrane phospholipids, as it

364 happens for Pro-7 and Leu-8 (35). In 1992, a biophysics work showed how Trp-49 and Trp-50 365 are not burying inside the bilayer when EGF and a membrane are in solution alone (36), though 366 this might be different when in complex with the receptor. For example, this could be achieved by Trp-50 through the formation of a helix, clustering together with Val-34 and Arg-45 around 
the conserved Leu-47 (37). Mutation N32R is on the interface between ligand and receptor.

The slightly higher affinity is probably due to the presence of the guanidinium group of $\mathrm{R}$ alter the mitogenicity without strongly affecting the binding affinity (38). While no previous literature reported about Asp-46 before, Lys-48 was found in two mutants that showed higher affinity (39). Our results give an explanation of the previous reports, while shedding new light on the role of the previously unreported Asp-46.

With the exception of N32R, all mutant ligands do not change in binding affinity to EGFR of compared to the wild type ligand. However, the mutants showed a different cellular effect on features that distinguish them from in vivo fibroblast, like differential gene expression and epiregulin-dependent proliferation (41). A decrease in cell proliferation after EGF treatment could be the result of competition between the endogenously synthesized epiregulin and EGF, thus altering the balance between proliferation and differentiation.

A431 cells constitutionally express EGFR at high levels. Treatment with EGF has been observed to promote STAT1 dependent apoptosis (42). This pathway is dependent on the internalization of the ligand-receptor complex by the endocytosis process, a key factor in the ligand-induced biased signaling (17). In our experiments, A431 cells treated with mutant EGF ligands show a higher growth rate, therefore a decreased rate of apoptosis compared to WT.

390 Given the setting of the experiment, modularity in the endocytosis pathway is a straightforward 391 explanation to the observed differences in growth rate and in protein levels and phosphorylation. Interestingly, K48T treated cells showed an increase in long and thin cellular 
393 processes in all replicates (Figure 5). This mutant was also observed with a markedly higher

394 EGFR phosphorylation $1 \mathrm{~h}$ after treatment (Figure 3). A possible explanation could be EGF-

395 induced tubular formation, an alternative EGF-induced pathway reported in intestinal epithelial

396 cells (43). In both the cellular growth experiments, the growth rate of the mutants is

397 intermediate between the control and EGF, and concentration dependent. This phenomenon,

398 and the cell-line specific response of growth hormones is consistent with previous literature

399 reports (44).

400

401 The observation that the four mutations in EGF alter signal transduction without disrupting any

402 contacts between the ligand and the receptor can be explained by the "loss of symmetry" model

403 of EGFR signaling (19). Freed et al. observed how stable symmetrical dimers show a short-

404 lived nature of signaling, while asymmetric dimers conduct a sustained activation, lasting

405 longer than $24 \mathrm{~h}$. Such a model provides an explanation on how a low level of phosphorylation

406 after EGF treatment causes the apoptotic behavior observed in the cellular growth experiments.

407 Compared with WT EGF, the K48T and W50Y variants show a higher phosphorylation signal,

408 suggesting a more sustained activation of EGFR, possibly through the formation of a less stable,

409 asymmetric dimer. Thus, the observed effects of the EGF mutations on EGFR phosphorylation

410 and dimer stability are consistent with the "loss of symmetry" mechanism.

412 The analysis of the fluctuations in the dimerization arm reveals an underlying bias in ligand-

413 induced receptor dimerization, originally not visible from the static images of structure

414 comparison. K48T is the mutant that induce the biggest deviation from the WT, in accordance

415 to the phosphorylation data. Although, all mutants show a transition. While giving an initial

416 outlook on the effect of the mutants, our MD simulations do not take into account other factors

417 that could be important in the mechanism of action of EGFR. Differential multimerization (45), 
receptor glycosylation, and the interaction with the membrane (23) are factors where the underlying bias induced by the mutant ligands could also have an effect in.

421 In this work, we showed how a single mutation of EGF is able to alter the specific functional relationship with the receptor. For functional divergence to arise, it could take as little as mutating $15 \%$ of the sequence (47). However, the EGFR ligands divergence date back to the vertebrate ancestor of R1/R2 whole genome duplication, up to 500 million years ago (48), as hinted by the low $(\sim 25 \%)$ sequence identity. From our results, the sequence distance does not reflect the distance in function. In fact, the functional divergence of EGF was altered with just a single targeted mutation.

In conclusion our data suggests that a single mutant ligand induces a conformational change of the receptor that then affects receptor dimer stability, with plausible effects on phosphorylation level and downstream pathway activation (49). This shows how the persistence of biased signaling in EGFR is in an unstable equilibrium, where the observed conservation of diverging sites among paralogs is naturally reinforced to maintain the functional divergence. To determine whether a short distance in function space among paralogs is a consequence or a necessity for living systems, further studies will be required.

Materials and Methods

\section{Sequence and structure analysis}

439 The sequences of EGFR ligands and the multiple sequence alignment of EGF orthologs were 440 obtained from the Ensembl database (51). Multiple sequence alignment (MSA) of all ligands 441 was performed with MAFFT software (52). X-ray structures were obtained from the PDB 442 database (53). Structural alignments were created using Chimera (54). 


\section{Phylogenetic analysis}

445 From the multiple sequence alignment of EGF from different species, nearly identical 446 sequences were removed. The Drosophila melanogaster EGF sequence was added as an 447 outgroup in the EGF phylogeny, while Caenorhabditis elegans EGF was used as outgroup for 448 the ligand phylogeny. MSA and phylogenetic tree images were created using Unipro UGENE 449 software (55). Three phylogenetic trees were made using the Neighbor Joining (NJ), Maximum 450 Likelihood (ML), and Bayesian (MrB) methods in IQTREE (56), using ModelFinder to scan 451 for the best-fit evolutionary model and parameters (57).

\section{Divergence Inducing Residue PREDiction}

454 We identified sites in EGF responsible for functional divergence using a method that combines evolutionary and co-evolutionary data, called DIRpred (Divergence Inducing Residue

456 PREDiction). The DIRpred scoring function combines four components to evaluate each

457 residue: I) The combined conservation scores in the ortholog alignments. This score is 458 calculated by averaging the conservation of a reference ligand site with the conservation in the 459 respective positions of the other ligands' orthologs alignments; II) The complementary value $460(1-\mathrm{x})$ of the conservation score in the paralogs alignment. This score can be obtained either 461 from sequence alignment (paralogs MSA) or structural alignment (paralogs MSTA). III) The 462 highest co-evolution score between a reference ligand site and all of the receptor sites. IV) The 463 complementary value $(1-\mathrm{x})$ of the highest co-evolution score between a reference ligand site 464 and all of the other ligand sites in the joint orthologs alignment of all ligands (Figure 7). 
1) Orthologs conservation

$I(p)=\frac{\sum_{l}^{\text {para }} \text { cons_score }\left(l_{p}\right)}{N_{l}}$

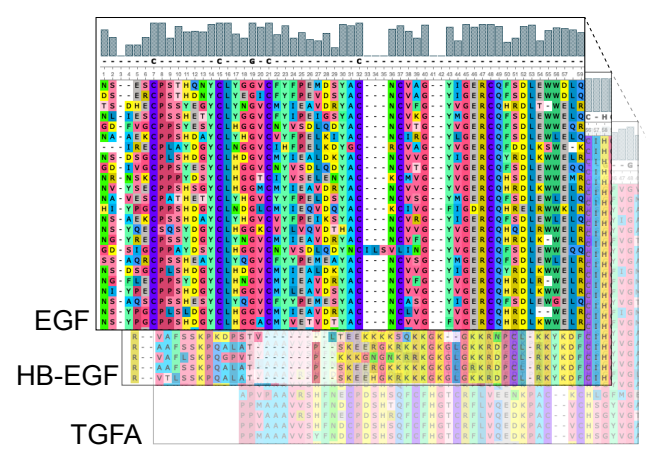

II ) Paralogs MSA/MSTA conservation

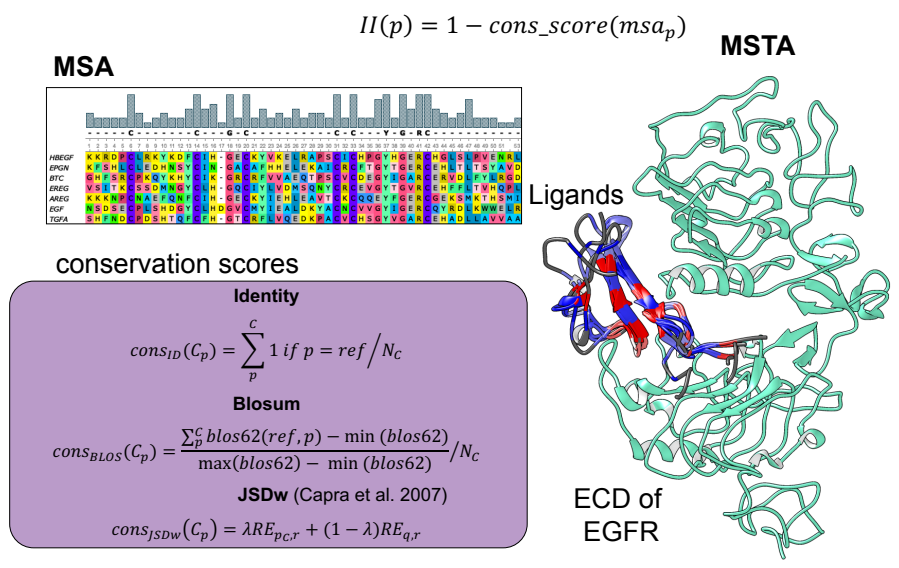

IV ) Ligands internal Co-evolution
III ) Ligand-receptor Co-evolution

$I I I(p)=\max \left(\right.$ coevo_score $\left.\left(r e f_{p}, E G F R_{*}\right)\right)$

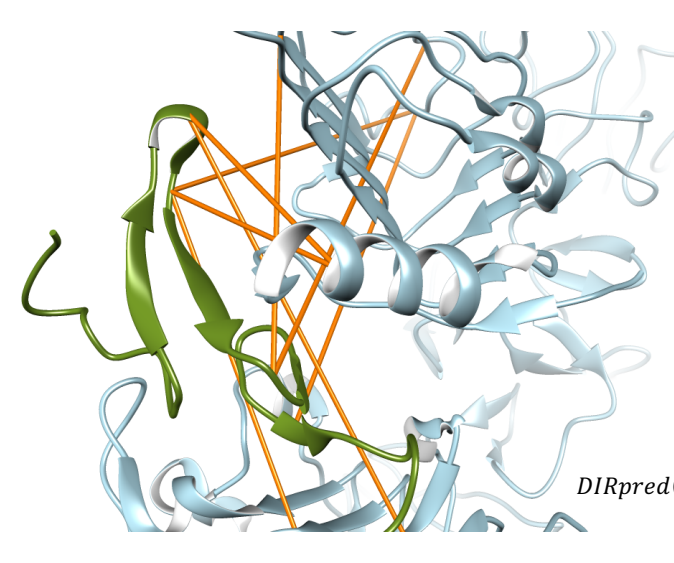

$I V(p)=1-\max \left(\right.$ coevo_score $\left(\right.$ para $_{p}$, para $\left.\left._{*}\right)\right)$

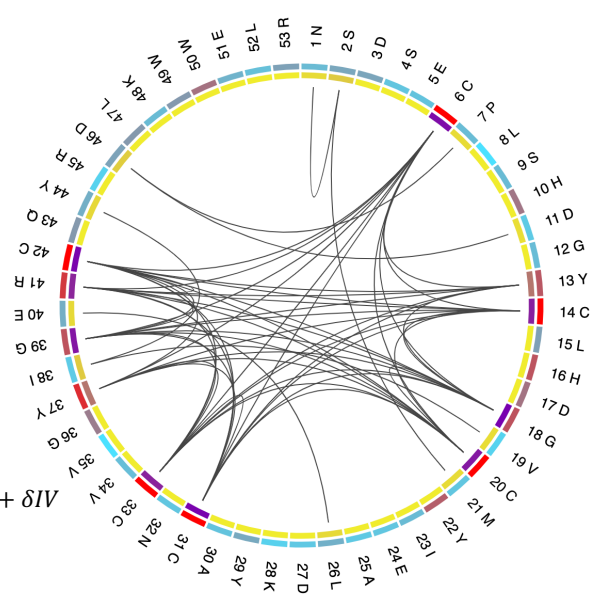

465

$$
\alpha+\beta+\gamma+\delta=1
$$

Figure 7. DIRpred rationale. The prediction of Divergence Inducing Residues consists of a

467 linear combination of four site-specific scores, in roman numerals. In I, the orthologs MSA of each paralog is used to compute a conservation score (as exemplified from the histograms on used. In the purple box, the conservation score functions are represented. In III, the coevolution score between EGF and each receptor site was computed using Mutual Information

473 (MI). The yellow lines on the structure connect the highest scoring co-evolving residues 474 between ligand and receptor. In IV, MI co-evolution score was computed from the combined 475 ligands orthologs alignment. The circular plot was performed using MISTIC2 (59). 
I) Orthologs conservation

7 ligands

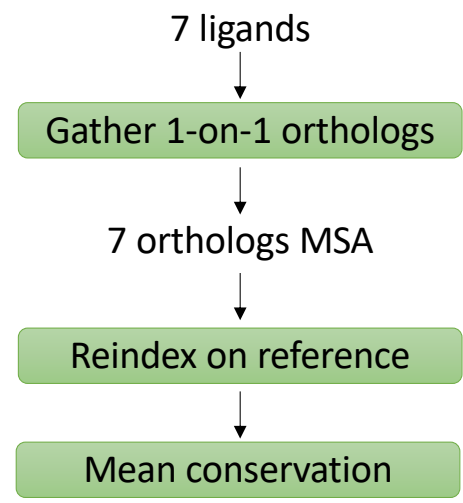

III) Ligand-Receptor Co-evolution

476

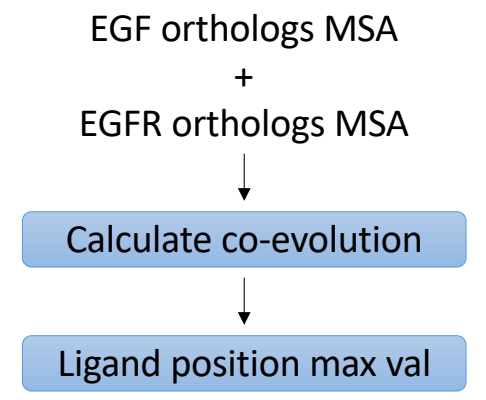

II) Paralogs MSA/MSTA conservation

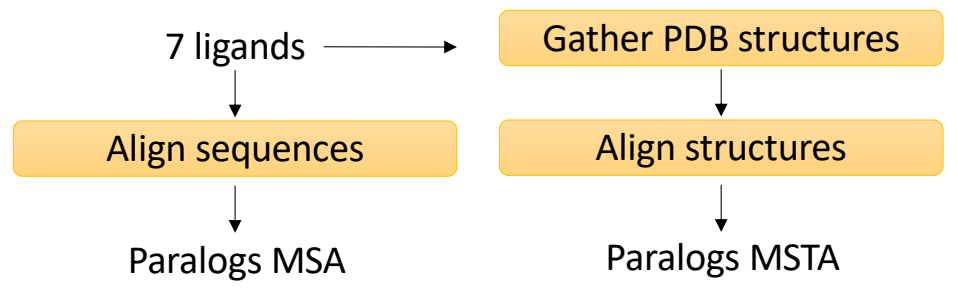

IV) Ligands internal Co-evolution

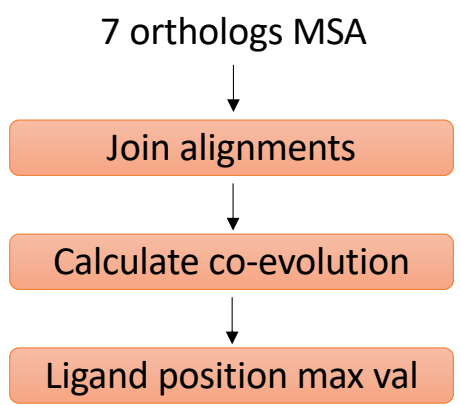

477 Figure 7-figure Supplement 1. DIRpred schema. A schematic representation of the

478 previous figure, showing the workflow to produce each of the four scores.

480 Conservation scores were calculated using three different formulations: 1) IDENTITY, 2)

481 BLOSUM, and 3) JSDw. The IDENTITY score measures the frequency of appearance of a 482 reference residue in the MSA. The BLOSUM score takes the amino acid substitution frequency 483 into account using the BLOSUM62 matrix, normalized by the maximum and minimum score 484 for the BLOSUM matrix. The JSDw score is based on Jensen Shannon divergence with a 485 window of residues (60). The analysis and plots were performed with Python3 package 486 SEABORN. EGF index positions from the paralogs MSA and MSTA were respectively used to align the MSA and MSTA based scores. 
488 The code used in the analysis of the DIRpred score and plots and the data used in this paper are shared on Github: https://github.com/oist/DIRpred.

490

\section{Selection of the mutations}

492 Along with the DIRpred score, the choice of positions for mutation was influenced by two

493 manually curated factors: the distance from the receptor and the amino acid variation among

494 ligand types. We designed mutations with the aim of inferring a transition to the amino acid 495 properties in sites where the ligands show a different pattern. Overlapping residues at a given 496 position were divided into two groups, based on an EGF-like and non EGF-like stabilization 497 of the receptor dimer. This property was previously shown to follow binding affinity (19). 498 Residues that introduced a noticeable shift in amino acid properties between the two groups 499 were selected. For example, position N32 is hydrophobic in the high affinity ligands group, 500 positively charged in the low affinity group and negatively charged in EGF. Finally, we 501 carefully analyzed exceptional cases in the DIRpred scoring. Some of the residues that show 502 high score have intramolecular interactions with another amino acid in the ligand. These residues, if mutated, will lose EGF structural stability (namely "residue swapping" behavior

504 showed in Figure 2-figure Supplement 3). The decision of which mutation to introduce was 505 made using the paralogs alignment. Positions 32, 48 and 50 have high DIRpred score. Position 50646 was included although having a lower score, because the substitution pattern matches the 507 two ligands groupings. Furthermore position 46, 48 and 50 were preferred because, given 508 previous experiments and the overall scores, the EGF C-terminus tail seems to play a critical 509 role in the ligand function, for example (61).

\section{Synthetic Peptides}


512 The wild-type, N32R, D46T, K48T, and W50Y variants of EGF were ordered from Scrum

513 Net Co with purity $>90 \%$ and quantity $5 \mathrm{mg} / \mathrm{mL}$. These peptides were used for ITC

514 measurements, Circular Dichroism (CD) measurements, proliferation studies, and Western

515 Blot (WB) analyses.

516

517 Cell Lines

518 The Bj5-ta human normal fibroblast cell line was purchased from ATCC. Cells were grown in

519 DMEM with $10 \%$ fetal bovine serum (FBS), and $5 \mu \mathrm{g} / \mathrm{mL}$ hygromycin B. The Swiss Albino

$5203 T 3$ mouse normal fibroblast cell line was obtained from the RIKEN Cell Bank. Cells were

521 grown in DMEM, $10 \% \mathrm{FBS}, 50 \mathrm{ug} / \mathrm{mL}$ gentamycin at $37^{\circ} \mathrm{C}$ in a $5 \% \mathrm{CO}_{2}$ atmosphere with $95 \%$

522 humidity. The A431 human epithelial carcinoma adherent cell line (RIKEN Cell Bank) is a

523 model skin cancer cell line with overexpressed EGFR used for oncogenic pathway studies

524 (62).Cells were cultured in DMEM supplemented with 10\% FBS (Sigma-Aldrich), $50 \mathrm{ug} / \mathrm{mL}$

525 gentamycin antibiotic or a combination of 100 unit/ml Penicillin G (Nacalai Tesque) and 100

$526 \mu \mathrm{g} / \mathrm{ml}$ streptomycin sulfate (Nacalai Tesque). Experiments were conducted at $37^{\circ} \mathrm{C}$ in a $5 \%$

$527 \mathrm{CO}_{2}$-enriched air atmosphere with $95 \%$ humidity. Cell lines were grown and used for Western

528 Blot and cell proliferation studies.

529

530 Cell Proliferation Assay

531 We measured cell proliferation using a label-free, non-invasive, cellular confluence assay with

532 IncuCyte Live-Cell Imaging Systems (Essen Bioscience). Human Bj5-ta (2,500 cells / well)

533 and Mouse Swiss Albino 3T3 (1,000 cells/well) were seeded overnight on a 96-well plate

534 (Corning) at $37^{\circ} \mathrm{C}$ in an incubator. The next day, cells were treated with WT EGF and mutants

535 at $1 \mathrm{nM}, 10 \mathrm{nM}$ and $100 \mathrm{nM}$ concentrations and placed in an XL-3 incubation chamber

536 maintained at $37^{\circ} \mathrm{C}$. The plate was scanned using a $4 \mathrm{x}$ objective at 2 -hr intervals over 3 days. 
537 Cell confluence was measured using IncuCyte Analysis Software. The IncuCyte Analyzer 538 gives real-time confluence data based on segmentation of high-definition phase-contrast 539 images. Cell proliferation is shown as an increase in confluence rate relative to control.

\section{Apoptosis Assay}

542 Experiments were performed with the A431 human cancer cell line. 5,000 cells/well were seeded on a 96-well plate (Corning) and incubated at $37^{\circ} \mathrm{C}$ for $24 \mathrm{hr}$. Media were replaced with fresh DMEM containing WT EGF, or EGF mutants at 1, 10, and $100 \mathrm{nM}$ concentrations and fluorescent annexin $\mathrm{V}$ green reagent. Plates were pre-warmed prior to data acquisition to avoid condensation and expansion of the plate, which affect autofocus. Images were captured every $2 \mathrm{hrs}(4 \mathrm{x})$ for 3 days in the IncuCyte system. Cell proliferation is reported as in the previous assay.

$550 \quad$ Statistics

551 Proliferation and apoptosis experiments were performed in duplicates. All results are shown as the mean \pm s.d. Raw data was analyzed by two-way ANOVA with 95\% confidence level. The multiple test was corrected using Bonferroni post hoc test. Prism 8 software was used

554 for statistical analysis. Asterisks in the pictures show P-values using GraphPad convention: $0.1234>(\mathrm{ns}), 0.0332>(*), 0.0021>(* *), 0.0002>(* * *), 0.0001>(* * * *)$.

\section{Isothermal Titration Calorimetry (ITC)}

558 All ITC studies employed a MicroCal PEAQ-ITC System (Malvern). For titration, both EGFR 559 ECD (Sigma-Aldrich) and EGF variants were dialyzed into the same reaction buffer Milli-Q $560 \mathrm{H}_{2} \mathrm{O}(22 \mu \mathrm{m})$ at $25^{\circ} \mathrm{C}$. Each titration involved serial injections of $13 \times 3 \mu \mathrm{L}$ aliquots of EGF 561 variants $(200 \mu \mathrm{M})$ into a solution of EGFR ECD $(20 \mu \mathrm{M})$ in the sample cell. In each case, the 
562 reference cell was filled with the same reaction buffer as the control to determine the heat upon

563 binding of the two components. The measured heat constant value was subtracted from the heat

564 per injection prior to analysis of the data. The experiment was replicated twice. Results were

565 analyzed by MicroCal PEAQ-ITC Analysis Software.

\section{Circular Dichroism (CD)}

568 Far UV measurements were taken at a protein concentration of $0.1 \mu \mathrm{M}$, using a cuvette with a 569 path length of $0.1 \mathrm{~cm}$. Secondary structure content was calculated from far UV spectra using 570 CAPITO software (27). Five scans in the 190-240-nm wavelength range were taken.

\section{Western Blot Analysis}

A431 epidermoid carcinoma cells were harvested using Lysis Buffer (0.4\% SDS, 1 mM DTT, $\mathrm{rpm}$ at $22^{\circ} \mathrm{C}$ for $10 \mathrm{~min}$. Supernatants were used for further analysis. Sample concentrations were measured with a BCA protein assay kit (ThermoFisher Scientific). Lysate were mixed with 4x Sample Loading Laemmli Buffer and incubated at $90^{\circ} \mathrm{C}$ for $5 \mathrm{~min}$. Equal amounts of protein were loaded in $12 \%$ Mini PROTEAN® ${ }^{\circledR}$ TGX ${ }^{\mathrm{TM}}$ SDS-PAGE gel (Bio-Rad) and transferred to PVDF membranes (Trans-Blot Turbo RTA Mini $0.2 \mu \mathrm{m}$ PVDF Transfer Kit).

580 Membranes were blocked for 10 min with Bullet Blocking One (Nacalai) and reacted with 581 monoclonal rabbit anti-EGFR antibody (Cell Signaling Technology, Inc.), Phospho-EGF Receptor (Tyr1173) (Cell Signaling Technology, Inc.), monoclonal rabbit anti-PLCy (Cell

583 Signaling Technology, Inc.), and anti-phosphorylated PLCy antibodies (Cell Signaling

584 Technology, Inc.), rabbit anti-PKC $\zeta$ (Cell Signaling Technology, Inc.), rabbit anti-phospho-

$585 \mathrm{PKC} \zeta / \lambda$ (Thr410/403) (Cell Signaling Technology, Inc.) and rabbit anti- $\alpha$-tubulin pAb (MBL) 
587 dilution and chemiluminescent signals were detected by CDP Plus (ThermoFisher Scientific)

588 and ChemiDoc touch MP (Bio-rad).

589

\section{$590 \quad$ Molecular Dynamics}

591 All the simulations were performed with Gromacs version 2020 (63) using charmm36-

592 mar2019 force field (64) and SPC216 for water. PDB ID 5wb7 was used as model and template.

593 EGF structure model was extracted from PDB ID 1ivo and superimposed to the ligand using

594 UCSF Chimera Match-Maker algorithm (65). Mutants were generated using Swissmodel

595 webserver (66), while missing atoms were compensated using Scwrl4 (67). A system was

596 composed of EGFR dimer in complex with one or two EGF wild type (WT), N32R, D46T,

$597 \mathrm{~K} 48 \mathrm{~T}$ or W50Y were solvated and neutralized using $\mathrm{NaCl}$ ions in a dodecahedral box. Then,

598 energy temperature and pressure equilibrations were performed to the system following the

599 guidelines in Lemkul 2019 (68).

600

601 A 100 ns production simulation was run using the Verlet cut-off scheme (69) for non-bonded

602 interactions and LINCS as constraint algorithm (70). All the simulations reached convergence

603 of RMSD. Long range electrostatic interactions were computed using the Particle Mesh Ewald 604 method (71) using a dedicated GPU. The parameter files for all runs are included in the 605 supplementary data [\#ADD\#]. We checked for differences in relative motions between the 606 three simulations by extracting and concatenating the backbone trajectories using catDCD 607 plugin of VMD (72), then performing a PCA using Bio3d R package (73).

608

609 Author contributions 
610 PL, SP, DM conceived and designed the project; SP developed and performed the

611 computational models and analyses; DM and GU performed the experiments; all authors

612 analyzed the data and wrote the manuscript.

613

\section{Acknowledgments}

615 We thank Tadashi Yamamoto, Ichiro Maruyama for providing the antibodies, Keiko Kono for

616 the technical help with the WB experiments. We are grateful for the help and support provided

617 by the Scientific Computing section of Research Support Division and in particular Jan Moren

618 at OIST. We thank Madhuri Gade, Mirco Dindo, Harry Wilson and Benjamin Clifton for

619 critical reading. We thank Pierre Matricon for the discussion on the molecular dynamics data,

620 Russel Alenton on the cell proliferation assay and Christine Guzman on the western blot

621 analyses.

623 Funding

624 Financial support by the Okinawa Institute of Science and Technology to P.L. is gratefully acknowledged.

\section{References}

628 1. Gazdar AF. Activating and resistance mutations of EGFR in non-small-cell lung 629 cancer: role in clinical response to EGFR tyrosine kinase inhibitors. Oncogene. 2009;28:S24630 S31.

631 2. Oda K, Matsuoka Y, Funahashi A, Kitano H. A comprehensive pathway map of epidermal growth factor receptor signaling. Mol Syst Biol. 2005;1:2005.0010.

3. Landau M, Fleishman SJ, Ben-Tal N. A putative mechanism for downregulation of the catalytic activity of the EGF receptor via direct contact between its kinase and C-terminal domains. Structure. 2004;12(12):2265-75.

4. Purba ER, Saita EI, Maruyama IN. Activation of the EGF Receptor by Ligand Binding and Oncogenic Mutations: The "Rotation Model". Cells. 2017;6(2).

638 5. Wilson KJ, Gilmore JL, Foley J, Lemmon MA, Riese DJ, 2nd. Functional selectivity 639 of EGF family peptide growth factors: implications for cancer. Pharmacol Ther. $640 \quad 2009 ; 122(1): 1-8$. 
641

642

643

644

645

646

647

648

649

650

651

652

653

654

655

656

657

658

659

660

661

662

663

664

665

666

667

668

669

670

671

672

673

674

675

676

677

678

679

680

681

682

683

684

685

686

687

688

6. Nicholson RI, Gee JM, Harper ME. EGFR and cancer prognosis. European journal of cancer (Oxford, England : 1990). 2001;37 Suppl 4:S9-15.

7. $\mathrm{Xu}$ MJ, Johnson DE, Grandis JR. EGFR-targeted therapies in the post-genomic era. Cancer Metastasis Rev. 2017;36(3):463-73.

8. Alvarez-Ponce D, Feyertag F, Chakraborty S. Position Matters: Network Centrality Considerably Impacts Rates of Protein Evolution in the Human Protein-Protein Interaction Network. Genome Biol Evol. 2017;9(6):1742-56.

9. Jensen RA. Orthologs and paralogs - we need to get it right. Genome Biol. 2001;2(8):Interactions1002.

10. Rao VS, Srinivas K, Sujini GN, Kumar GN. Protein-protein interaction detection: methods and analysis. International journal of proteomics. 2014;2014:147648.

11. Groenen LC, Nice EC, Burgess AW. STRUCTURE-FUNCTION-RELATIONSHIPS FOR THE EGF/TGF-ALPHA FAMILY OF MITOGENS. Growth Factors. 1994;11(4):23557.

12. Souriau C, Gracy J, Chiche L, Weill M. Direct selection of EGF mutants displayed on filamentous phage using cells overexpressing EGF receptor. Biol Chem. 1999;380(4):451-8.

13. Skwark MJ, Raimondi D, Michel M, Elofsson A. Improved Contact Predictions Using the Recognition of Protein Like Contact Patterns. PLOS Computational Biology. 2014;10(11):e1003889.

14. Gocheva G, Ivanova A. A Look at Receptor-Ligand Pairs for Active-Targeting Drug Delivery from Crystallographic and Molecular Dynamics Perspectives. Mol Pharm. 2019;16(8):3293-321.

15. Evans BA, Sato M, Sarwar M, Hutchinson DS, Summers RJ. Ligand-directed signalling at beta-adrenoceptors. Br J Pharmacol. 2010;159(5):1022-38.

16. Kenakin T. Functional selectivity and biased receptor signaling. J Pharmacol Exp Ther. 2011;336(2):296-302.

17. Ali R, Brown W, Purdy SC, Davisson VJ, Wendt MK. Biased signaling downstream of epidermal growth factor receptor regulates proliferative versus apoptotic response to ligand. Cell Death Dis. 2018;9(10):976.

18. Strachan L, Murison JG, Prestidge RL, Sleeman MA, Watson JD, Kumble KD. Cloning and biological activity of epigen, a novel member of the epidermal growth factor superfamily. The Journal of biological chemistry. 2001;276(21):18265-71.

19. Freed DM, Bessman NJ, Kiyatkin A, Salazar-Cavazos E, Byrne PO, Moore JO, et al. EGFR Ligands Differentially Stabilize Receptor Dimers to Specify Signaling Kinetics. Cell. 2017;171(3):683-95.e18.

20. Mehrabi M, Mahdiuni H, Rasouli H, Mansouri K, Shahlaei M, Khodarahmi R. Comparative experimental/theoretical studies on the EGFR dimerization under the effect of EGF/EGF analogues binding: Highlighting the importance of EGF/EGFR interactions at site III interface. Int J Biol Macromol. 2018;115:401-17.

21. Knudsen SL, Mac AS, Henriksen L, van Deurs B, Grovdal LM. EGFR signaling patterns are regulated by its different ligands. Growth Factors. 2014;32(5):155-63.

22. Liu P, Cleveland TEt, Bouyain S, Byrne PO, Longo PA, Leahy DJ. A single ligand is sufficient to activate EGFR dimers. Proc Natl Acad Sci U S A. 2012;109(27):10861-6.

23. Arkhipov A, Shan Y, Kim ET, Shaw DE. Membrane interaction of bound ligands contributes to the negative binding cooperativity of the EGF receptor. PLoS Comput Biol. 2014;10(7):e1003742.

24. Chagoyen M, García-Martín JA, Pazos F. Practical analysis of specificity-determining residues in protein families. Briefings in Bioinformatics. 2015;17(2):255-61. 
25. Cochran JR, Kim YS, Lippow SM, Rao B, Wittrup KD. Improved mutants from directed evolution are biased to orthologous substitutions. Protein Eng Des Sel. 2006;19(6):245-53.

26. Prestrelski SJ, Arakawa T, Wu CS, O'Neal KD, Westcott KR, Narhi LO. Solution structure and dynamics of epidermal growth factor and transforming growth factor alpha. The Journal of biological chemistry. 1992;267(1):319-22.

27. Wiedemann C, Bellstedt P, Gorlach M. CAPITO--a web server-based analysis and plotting tool for circular dichroism data. Bioinformatics. 2013;29(14):1750-7.

28. Gill GN, Lazar CS. Increased phosphotyrosine content and inhibition of proliferation in EGF-treated A431 cells. Nature. 1981;293(5830):305-7.

29. Gulli LF, Palmer KC, Chen YQ, Reddy KB. Epidermal growth factor-induced apoptosis in A431 cells can be reversed by reducing the tyrosine kinase activity. Cell Growth Differ. 1996;7(2):173-8.

30. Nemoto W, Saito A, Oikawa H. Recent advances in functional region prediction by using structural and evolutionary information - Remaining problems and future extensions. Computational and structural biotechnology journal. 2013;8:e201308007.

31. Ashkenazy H, Abadi S, Martz E, Chay O, Mayrose I, Pupko T, et al. ConSurf 2016: an improved methodology to estimate and visualize evolutionary conservation in macromolecules. Nucleic Acids Res. 2016;44(W1):W344-W50.

32. Lichtarge O, Bourne HR, Cohen FE. An evolutionary trace method defines binding surfaces common to protein families. J Mol Biol. 1996;257(2):342-58.

33. Teppa E, Wilkins AD, Nielsen M, Buslje CM. Disentangling evolutionary signals: conservation, specificity determining positions and coevolution. Implication for catalytic residue prediction. BMC Bioinformatics. 2012;13(1):235.

34. Gallay J, Vincent M, Li de la Sierra IM, Alvarez J, Ubieta R, Madrazo J, et al. Protein flexibility and aggregation state of human epidermal growth factor. A time-resolved fluorescence study of the native protein and engineered single-tryptophan mutants. Eur J Biochem. 1993;211(1-2):213-9.

35. Tynan CJ, Roberts SK, Rolfe DJ, Clarke DT, Loeffler HH, Kastner J, et al. Human epidermal growth factor receptor (EGFR) aligned on the plasma membrane adopts key features of Drosophila EGFR asymmetry. Mol Cell Biol. 2011;31(11):2241-52.

36. Li De La Sierra IM, Vincent M, Padron G, Gallay J. Interaction of recombinant human epidermal growth factor with phospholipid vesicles. A steady-state and time-resolved fluorescence study of the bis-tryptophan sequence (TRP49-TRP50). European Biophysics Journal. 1992;21(5):337-44.

37. Hommel U, Harvey TS, Driscoll PC, Campbell ID. Human epidermal growth factor. High resolution solution structure and comparison with human transforming growth factor alpha. J Mol Biol. 1992;227(1):271-82.

38. Puddicombe SM, Chamberlin SG, MacGarvie J, Richter A, Drummond DR, Collins J, et al. The significance of valine 33 as a ligand-specific epitope of transforming growth factor alpha. The Journal of biological chemistry. 1996;271(26):15367-72.

39. Lahti JL, Lui BH, Beck SE, Lee SS, Ly DP, Longaker MT, et al. Engineered epidermal growth factor mutants with faster binding on-rates correlate with enhanced receptor activation. FEBS letters. 2011;585(8):1135-9.

40. Carpenter G, Cohen S. Epidermal growth factor. Annual review of biochemistry. 1979;48:193-216.

41. Lindvall C, Hou M, Komurasaki T, Zheng C, Henriksson M, Sedivy JM, et al. Molecular characterization of human telomerase reverse transcriptase-immortalized human fibroblasts by gene expression profiling: activation of the epiregulin gene. Cancer Res. 2003;63(8):1743-7. 

apoptosis in A431 cells is dependent on STAT1, but not on STAT3. Eur J Cell Biol. 2007;86(10):591-603.

43. Ibuka S, Matsumoto S, Fujii S, Kikuchi A. The P2Y(2) receptor promotes Wnt3a- and EGF-induced epithelial tubular formation by IEC6 cells by binding to integrins. J Cell Sci. 2015;128(11):2156-68.

44. Bjorkelund H, Gedda L, Andersson K. Comparing the epidermal growth factor interaction with four different cell lines: intriguing effects imply strong dependency of cellular context. PloS one. 2011;6(1):e16536.

748 45. Macdonald-Obermann JL, Pike LJ. Different epidermal growth factor (EGF) receptor ligands show distinct kinetics and biased or partial agonism for homodimer and heterodimer formation. The Journal of biological chemistry. 2014;289(38):26178-88.

751 46. Innan H, Kondrashov F. The evolution of gene duplications: classifying and distinguishing between models. Nature Reviews Genetics. 2010;11(2):97-108.

47. Zhou J, Liu D, Sa Z, Huang W, Zou Y, Gu X. Effective estimation of the minimum number of amino acid residues required for functional divergence between duplicate genes. Mol Phylogenet Evol. 2017;113:126-38.

48. Laisney J, Braasch I, Walter RB, Meierjohann S, Schartl M. Lineage-specific coevolution of the Egf receptor/ligand signaling system. BMC Evol Biol. 2010;10:16.

49. Bakker J, Spits M, Neefjes J, Berlin I. The EGFR odyssey - from activation to destruction in space and time. Journal of Cell Science. 2017;130(24):4087-96.

50. Sotelo MJ, García-Paredes B, Aguado C, Sastre J, Díaz-Rubio E. Role of cetuximab in first-line treatment of metastatic colorectal cancer. World journal of gastroenterology. 2014;20(15):4208-19.

51. Zerbino DR, Achuthan P, Akanni W, Amode MR, Barrell D, Bhai J, et al. Ensembl 2018. Nucleic Acids Res. 2018;46(D1):D754-D61.

52. Katoh K, Standley DM. MAFFT Multiple Sequence Alignment Software Version 7: Improvements in Performance and Usability. Mol Biol Evol. 2013;30(4):772-80.

53. Berman HM, Westbrook J, Feng Z, Gilliland G, Bhat TN, Weissig H, et al. The Protein Data Bank. Nucleic Acids Res. 2000;28(1):235-42.

54. Goddard TD, Huang CC, Meng EC, Pettersen EF, Couch GS, Morris JH, et al. UCSF ChimeraX: Meeting modern challenges in visualization and analysis. Protein Sci. 2018;27(1):14-25.

55. Okonechnikov K, Golosova O, Fursov M, Team U. Unipro UGENE: a unified bioinformatics toolkit. Bioinformatics. 2012;28(8):1166-7.

56. Nguyen LT, Schmidt HA, von Haeseler A, Minh BQ. IQ-TREE: A Fast and Effective Stochastic Algorithm for Estimating Maximum-Likelihood Phylogenies. Mol Biol Evol. 2015;32(1):268-74.

57. Kalyaanamoorthy S, Minh BQ, Wong TKF, von Haeseler A, Jermiin LS. ModelFinder: fast model selection for accurate phylogenetic estimates. Nat Methods. 2017;14(6):587-+.

58. Okonechnikov K, Golosova O, Fursov M, team tU. Unipro UGENE: a unified bioinformatics toolkit. Bioinformatics. 2012;28(8):1166-7.

781 59. Colell EA, Iserte JA, Simonetti FL, Marino-Buslje C. MISTIC2: comprehensive server to study coevolution in protein families. Nucleic Acids Res. 2018;46(W1):W323-w8.

60. Capra JA, Singh M. Predicting functionally important residues from sequence conservation. Bioinformatics. 2007;23(15):1875-82.

61. Puddicombe SM, Wood L, Chamberlin SG, Davies DE. The interaction of an epidermal growth factor/transforming growth factor alpha tail chimera with the human epidermal growth factor receptor reveals unexpected complexities. The Journal of biological chemistry. 1996;271(48):30392-7. 
62. Giard DJ, Aaronson SA, Todaro GJ, Arnstein P, Kersey JH, Dosik H, et al. In vitro cultivation of human tumors: establishment of cell lines derived from a series of solid tumors. J Natl Cancer Inst. 1973;51(5):1417-23.

792 63. Abraham MJ, Murtola T, Schulz R, Páll S, Smith JC, Hess B, et al. GROMACS: High performance molecular simulations through multi-level parallelism from laptops to supercomputers. SoftwareX. 2015;1-2:19-25. 64. Huang J, MacKerell AD, Jr. CHARMM36 all-atom additive protein force field: validation based on comparison to NMR data. J Comput Chem. 2013;34(25):2135-45. 65. Meng EC, Pettersen EF, Couch GS, Huang CC, Ferrin TE. Tools for integrated sequence-structure analysis with UCSF Chimera. BMC Bioinformatics. 2006;7:339.

799 66. Waterhouse A, Bertoni M, Bienert S, Studer G, Tauriello G, Gumienny R, et al. 800 SWISS-MODEL: homology modelling of protein structures and complexes. Nucleic Acids Res. $801 \quad 2018 ; 46(\mathrm{~W} 1): \mathrm{W} 296-\mathrm{W} 303$.

802 67. Krivov GG, Shapovalov MV, Dunbrack RL, Jr. Improved prediction of protein side803 chain conformations with SCWRL4. Proteins. 2009;77(4):778-95.

804 68. Lemkul JA. From Proteins to Perturbed Hamiltonians: A Suite of Tutorials for the 805 GROMACS-2018 Molecular Simulation Package [Article v1.0]. Living J Comp Mol Sci. $806 \quad 2019 ; 1(1): 5068$.

807 69. Páll S, Hess B. A flexible algorithm for calculating pair interactions on SIMD 808 architectures. Computer Physics Communications. 2013;184(12):2641-50.

809 70. Hess B, Bekker H, Berendsen HJC, Fraaije JGEM. LINCS: A linear constraint solver 810 for molecular simulations. Journal of Computational Chemistry. 1997;18(12):1463-72.

811 71. Essmann U, Perera L, Berkowitz ML, Darden T, Lee H, Pedersen LG. A smooth 812 particle mesh Ewald method. The Journal of Chemical Physics. 1995;103(19):8577-93.

813 72. Humphrey W, Dalke A, Schulten K. VMD: visual molecular dynamics. J Mol Graph. 814 1996;14(1):33-8, 27-8.

815 73. Grant BJ, Rodrigues AP, ElSawy KM, McCammon JA, Caves LS. Bio3d: an R package 816 for the comparative analysis of protein structures. Bioinformatics. 2006;22(21):2695-6. 


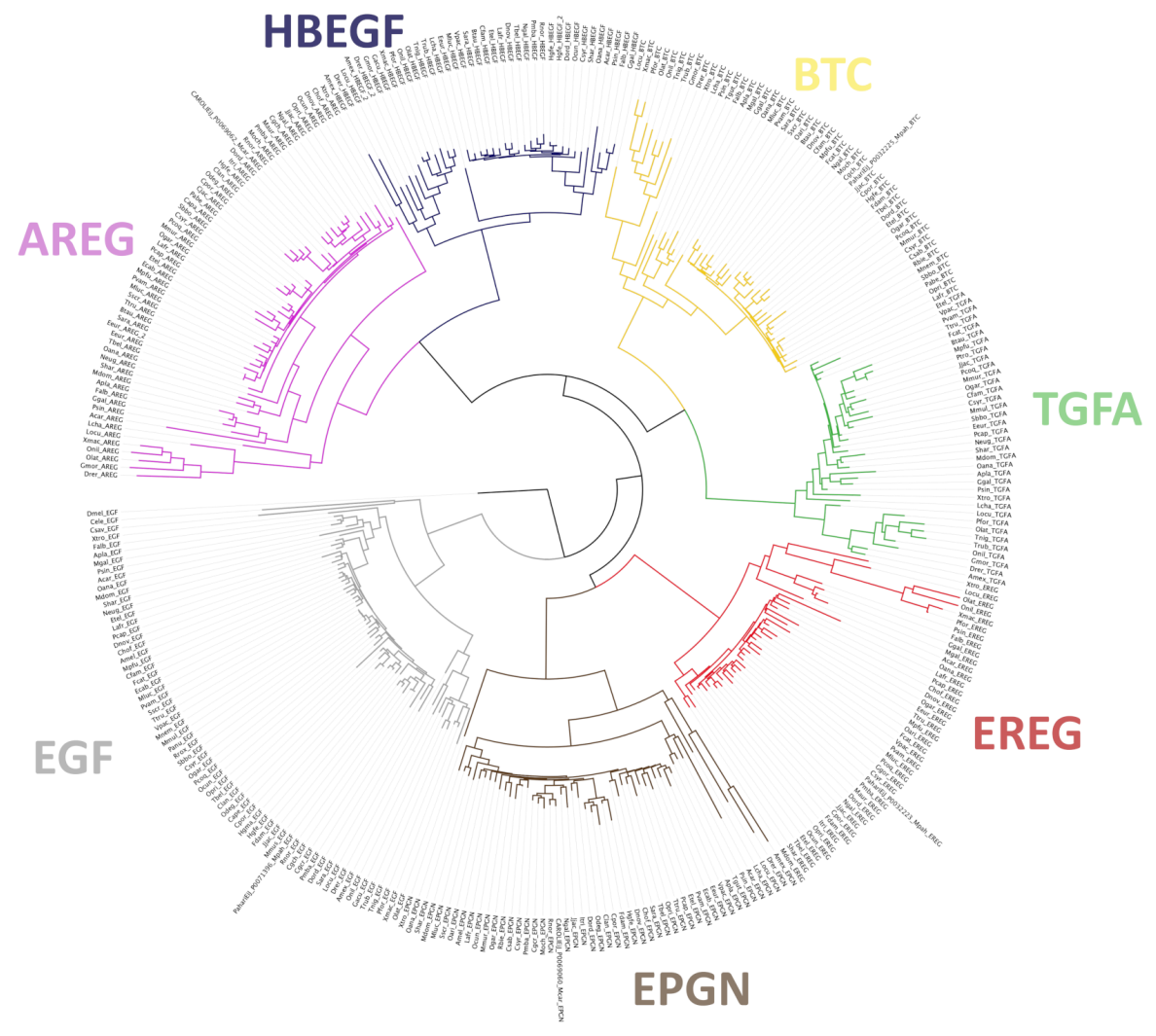

820 Supplementary figure 1. EGFR ligands orthologs tree. The tree was obtained using

821 Maximum Likelihood algorithm. C elegans EGF was used as outgroup. The EGFR ligands

822 cluster neatly. Interestingly, fish ligands are found to have longer branches on average. The

823 tree was made using iqtree ModelFinder. The best-fit model used is JTT+R6. 


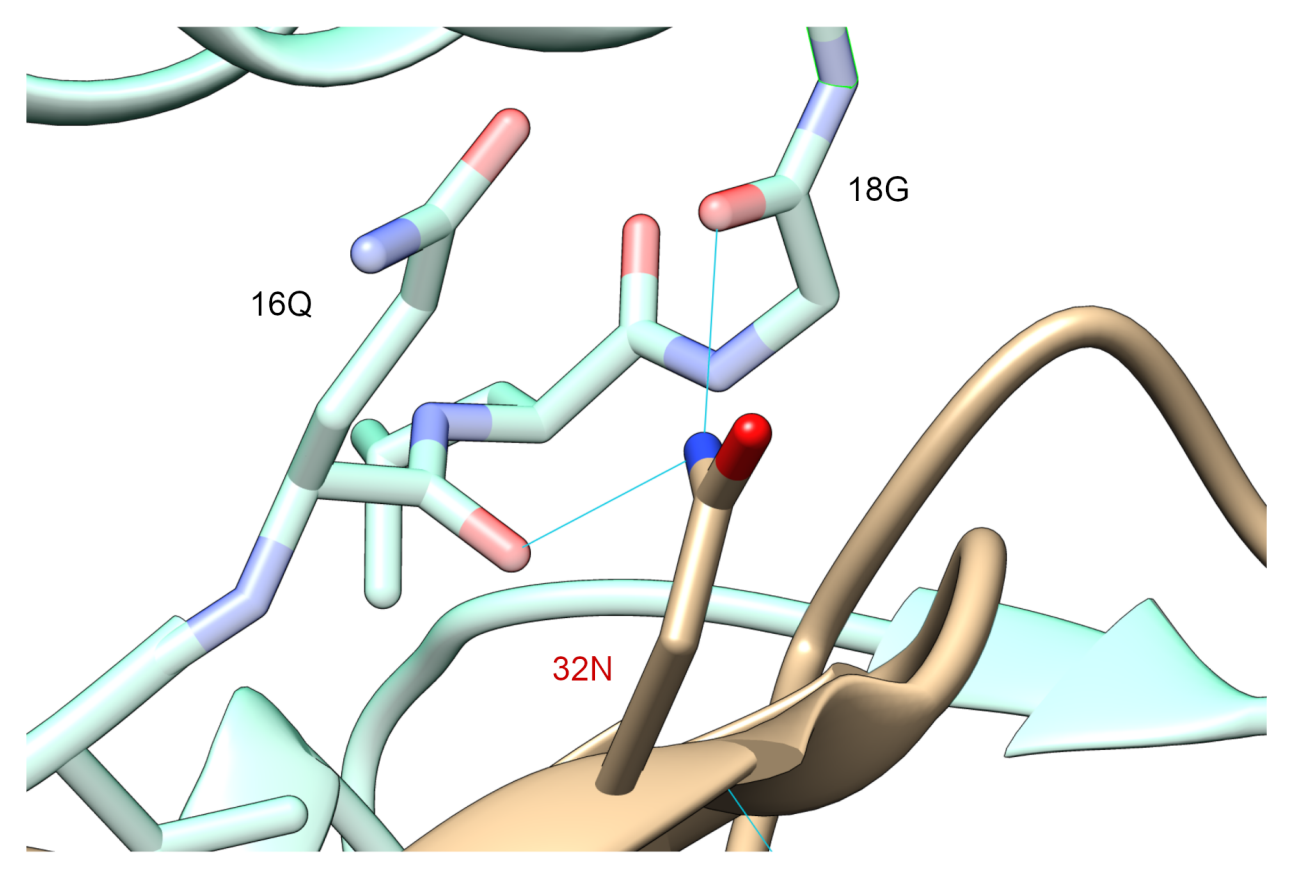

824

825 Supplementary figure 2. Crystalized structure of WT EGF with the highlighted position

$826 \quad$ N32, In the interaction with the position Q16 and G18 of the EGFR receptor backbone.

\section{A431 Human Cancer Cell Growth}

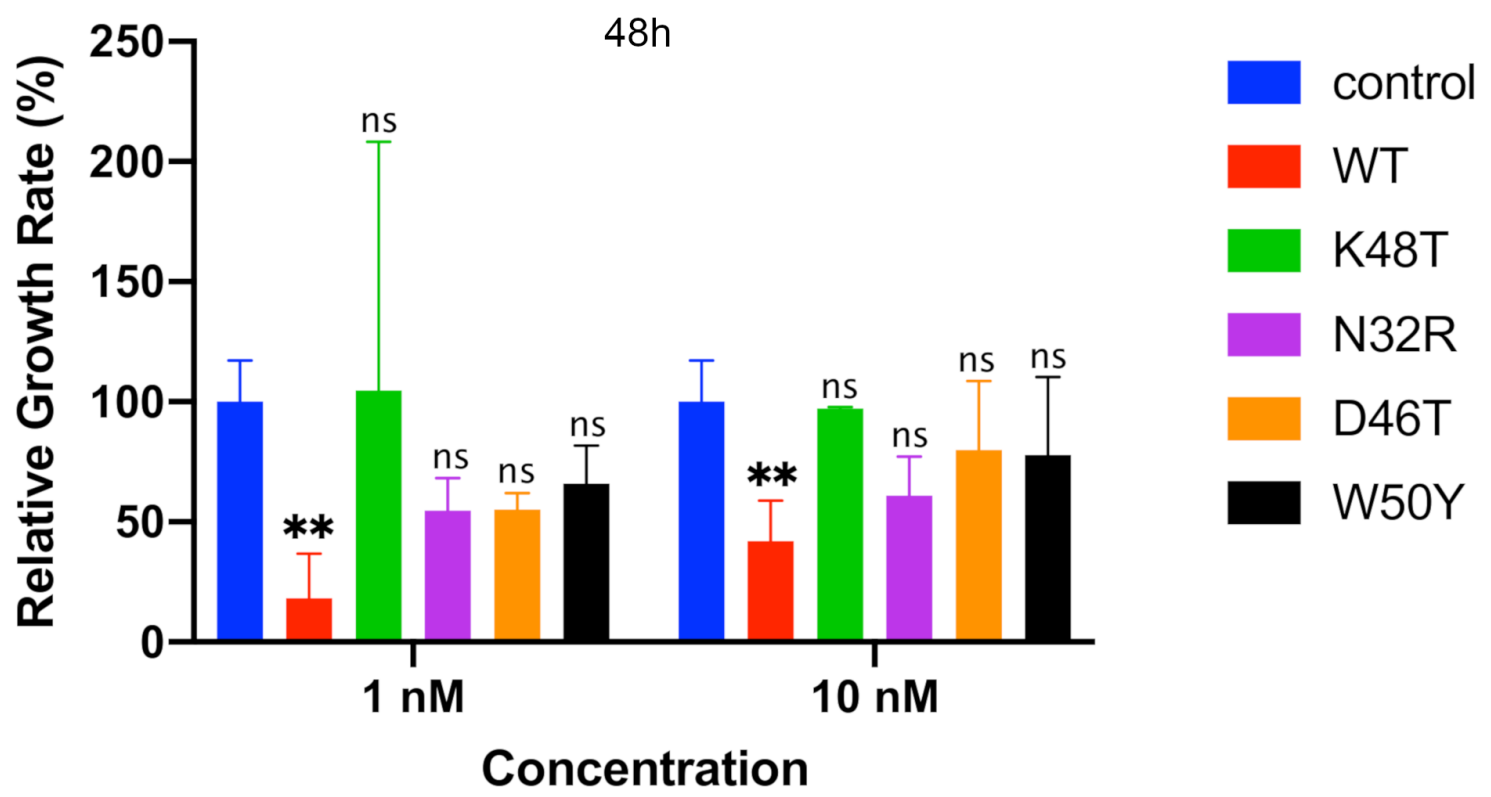


828 Supplementary figure 3. Effect of $1 \mathrm{nM}$ and $10 \mathrm{nM}$ concentration of EGF variants on the

829 proliferation of A431 skin cancer cells. The data represent the relative growth percentage to

830 control based on the confluence of cells (mean \pm standard deviation) for each concentration of

831 EGF variants. The percentage confluence was estimated $48 \mathrm{~h}$ after the treatment (two

832 replicates/treatment).

833

834 Supplementary data 1. Time-lapse video of the proliferation of A431 and Bj-5t $\alpha$ cells.

835 Treatment with $100 \mathrm{nM}$ ligand was performed at the $24 \mathrm{~h}$ mark. Evidences of a balance between

836 differentiation and proliferation are found in the fibroblast, where a reduced proliferation is

837 associated to cellular morphology differences in WT and K48T.

838 